\title{
Poset pinball, highest forms, and $(n-2,2)$ Springer varieties
}

\author{
Barry Dewitt \\ Mathematical Institute \\ University of Oxford \\ Oxford, U.K. \\ barry.dewitt@maths.ox.ac.uk
}

\author{
Megumi Harada* \\ Department of Mathematics and Statistics \\ McMaster University \\ Hamilton, Canada \\ Megumi.Harada@math.mcmaster .ca
}

Submitted: Jan 7, 2011; Accepted: Mar 8, 2012; Published: Mar 19, 2012
Mathematics Subject Classifications: 14M17; 55N91

\begin{abstract}
In this manuscript we study type $A$ nilpotent Hessenberg varieties equipped with a natural $S^{1}$-action using techniques introduced by Tymoczko, Harada-Tymoczko, and Bayegan-Harada, with a particular emphasis on a special class of nilpotent Springer varieties corresponding to the partition $\lambda=(n-2,2)$ for $n \geq 4$. First we define the adjacent-pair matrix corresponding to any filling of a Young diagram with $n$ boxes with the alphabet $\{1,2, \ldots, n\}$. Using the adjacent-pair matrix we make more explicit and also extend some statements concerning highest forms of linear operators in previous work of Tymoczko. Second, for a nilpotent operator $N$ and Hessenberg function $h$, we construct an explicit bijection between the $S^{1}$-fixed points of the nilpotent Hessenberg variety $\operatorname{Hess}(N, h)$ and the set of $\left(h, \lambda_{N}\right)$-permissible fillings of the Young diagram $\lambda_{N}$. Third, we use poset pinball, the combinatorial game introduced by Harada and Tymoczko, to study the $S^{1}$-equivariant cohomology of type $A$ Springer varieties $\mathcal{S}_{(n-2,2)}$ associated to Young diagrams of shape $(n-2,2)$ for $n \geq 4$. Specifically, we use the dimension pair algorithm for Betti-acceptable pinball described by Bayegan and Harada to specify a subset of the equivariant Schubert classes in the $\mathbb{T}$-equivariant cohomology of the flag variety $\mathcal{F}$ lags $\left(\mathbb{C}^{n}\right) \cong G L(n, \mathbb{C}) / B$ which maps to a module basis of $H_{S^{1}}^{*}\left(\mathcal{S}_{(n-2,2)}\right)$ under the projection map $H_{\mathbb{T}}^{*}\left(\mathcal{F l a g s}\left(\mathbb{C}^{n}\right)\right) \rightarrow H_{S^{1}}^{*}\left(\mathcal{S}_{(n-2,2)}\right)$. Our poset pinball module basis is not poset-upper-triangular; this is the first concrete such example in the literature. A straightforward consequence of our proof is that there exists a simple and explicit change of basis which transforms our poset pinball basis to a posetupper-triangular module basis for $H_{S^{1}}^{*}\left(\mathcal{S}_{(n-2,2)}\right)$. We close with open questions for future work.
\end{abstract}

*Partially supported by an NSERC Discovery Grant, an NSERC University Faculty Award, and an Ontario Ministry of Research and Innovation Early Researcher Award. 


\section{Contents}

1 Introduction $\quad 2$

2 Nilpotent Hessenberg varieties and $S^{1}$-actions $\quad 5$

3 Adjacent-pair matrices and highest forms of nilpotent operators $\quad 6$

$4 \quad S^{1}$-fixed points in Hessenberg varieties and permissible fillings $\quad 17$

5 Betti-acceptable pinball and linear independence $\quad 20$

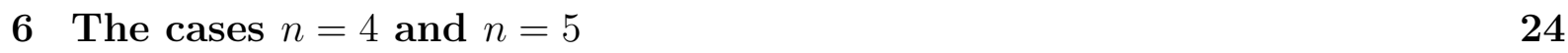

$7 \quad$ A poset pinball module basis for $(n-2,2)$ Springer varieties $\quad 27$

8 Open questions $\quad 33$

\section{Introduction}

The study of Hessenberg varieties is an active field of modern mathematical research. Indeed, Hessenberg varieties arise in many areas of mathematics, including geometric representation theory $[8,15,16]$, numerical analysis [6], mathematical physics [12, 14], combinatorics [7], and algebraic geometry [4,5], so it is of interest to explicitly analyze their topology, e.g. the structure of their (equivariant) cohomology rings. In this paper we further develop the approach, initiated and developed in $[1,2,9,10]$, which studies the topology of Hessenberg varieties through poset pinball and Schubert calculus techniques.

In this manuscript we focus on the case of nilpotent Hessenberg varieties, and more particularly on nilpotent Springer varieties. We begin by briefly recalling the setting of our results; for more details we refer the reader to Section 2 . Let $N: \mathbb{C}^{n} \rightarrow \mathbb{C}^{n}$ be a nilpotent operator. Let $h:\{1,2, \ldots, n\} \rightarrow\{1,2, \ldots, n\}$ be a function satisfying $h(i) \geq i$ for all $1 \leq i \leq n$ and $h(i+1) \geq h(i)$ for all $1 \leq i<n$. In type $A$, nilpotent Hessenberg varieties can be defined as the following subvariety of $\mathcal{F}$ lags $\left(\mathbb{C}^{n}\right)$ :

$$
\begin{aligned}
\operatorname{Hess}(N, h):=\left\{V_{\bullet}=(0 \subseteq\right. & V_{1} \subseteq V_{2} \subseteq \\
& \left.\left.\cdots \subseteq V_{n-1} \subseteq V_{n}=\mathbb{C}^{n}\right) \mid N V_{i} \subseteq V_{h(i)} \text { for all } i=1, \ldots, n\right\} .
\end{aligned}
$$

We equip $\operatorname{Hess}(N, h)$ with a natural $S^{1}$-action (described precisely in Section 2) induced from the diagonal torus subgroup $\mathbb{T}$ of $U(n, \mathbb{C})$ acting in the usual fashion on $G L(n, \mathbb{C}) / B \cong \mathcal{F l a g s}\left(\mathbb{C}^{n}\right)$. In the special case when the Hessenberg function $h:\{1,2, \ldots, n\} \rightarrow\{1,2, \ldots, n\}$ is the identity $h(i)=i$ for all $1 \leq i \leq n$, we call $\operatorname{Hess}(N, h)$ a nilpotent Springer variety and denote it by $\mathcal{S}_{N}$.

Our first results apply to general type $A$ nilpotent Hessenberg varieties. Let $N$ be a nilpotent $n \times n$ matrix in Jordan canonical form with weakly decreasing block sizes and 
let $\lambda$ denote the Young diagram ${ }^{1}$ (equivalently the partition) with row lengths the Jordan block sizes of $N$ listed in weakly decreasing order. In [17, Theorem 6.1] Tymoczko builds a paving-by-affines of a nilpotent Hessenberg variety $\operatorname{Hess}(N, h)$, where the nilpotent operator $N$ is required to be in a highest form (see [17, Definition 4.1]). Much topological information about a variety is encoded in a paving-by-affines, so it is useful to build tools for dealing with the technical condition that the operator $N$ be in a highest form. We introduce what we call the adjacency-pair matrix, which is an $n \times n$ matrix constructed from a filling of a Young diagram $\lambda$ with $n$ boxes by the alphabet $\{1,2, \ldots, n\}$. This then allows us to make more explicit and also generalizes a procedure for producing highest forms of linear operators sketched in [17, Section 4]. In particular our methods allow us to straightforwardly derive the explicit change-of-basis permutation matrix which puts $N$ into any choice of a highest form (including that used by Tymoczko in [17], cf. Corollary 3.23). The adjacent-pair matrices also allows us to see precisely the set of permutation matrices which conjugate $N$ to a highest form (Theorem 3.21). The explicit nature of our results allows for other computations related to these nilpotent Hessenberg varieties. As an example, we derive in Lemma 3.29 an explicit formula for the Lie algebra projection induced by the inclusion of the $S^{1}$ subgroup acting on a special case of nilpotent Springer variety into the diagonal subgroup $\mathbb{T}$ of $U(n, \mathbb{C})$ acting on $\mathcal{F}$ lags $\left(\mathbb{C}^{n}\right)$. Thus we expect our procedure to be useful for future poset pinball analysis of type $A$ nilpotent Hessenberg varieties.

The affine cells in Tymoczko's paving-by-affines of $\operatorname{Hess}(N, h)$ are in one-to-one correspondence with permissible fillings of Young diagrams (defined precisely in Section 4); this is a useful combinatorial enumeration of the affine cells. The correspondence arises since the affine cells are intersections of $\operatorname{Hess}(N, h)$ with certain Schubert cells $B w B \subseteq G L(n, \mathbb{C}) / B \cong \mathcal{F}$ lags $\left(\mathbb{C}^{n}\right)$. Each such Schubert cell contains a unique (coset of a) permutation matrix $w B$, and each permutation $w$ can be associated to a permissible filling of $\lambda$. We extend this relationship between the permutations (which in this manuscript we think of as $S^{1}$-fixed points of $\operatorname{Hess}(N, h)$ ) and the permissible fillings as follows. For the purpose of the discussion below assume that $N$ is in Jordan canonical form with weakly decreasing block sizes. We define for each permutation $\sigma \in S_{n}$ a bijection $\phi_{\lambda, \sigma}$ between the set $\mathcal{F} i \ell \ell(\lambda)$ of fillings of $\lambda$ with the set of permutations $S_{n}$ (Definition 4.3). Each $\phi_{\lambda, \sigma}$ then induces a bijection between the permissible fillings $\mathcal{P} F i \ell \ell(\lambda)$ of $\lambda$ and the $S^{1}$-fixed points of the translated Hessenberg variety Hess $\left(\sigma N \sigma^{-1}, h\right)$ (cf. Theorem 4.7). Our results also provide proofs of statements quoted in [2].

Finally, we give an explicit construction of a computationally convenient module basis for the $S^{1}$-equivariant cohomology ${ }^{2}$ of a special class of type $A$ nilpotent Springer varieties, namely, the 2-block (also known as 2-row) nilpotent Springer varieties associated to Young diagrams of the form $(n-2,2)$, e.g.

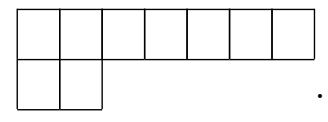

\footnotetext{
${ }^{1}$ We use English notation for Young diagrams.

${ }^{2}$ We work with cohomology with coefficients in $\mathbb{C}$ throughout, and hence omit it from our notation.
} 
The motivation for our construction comes from (equivariant) geometry, and more specifically from generalized Schubert calculus and geometric representation theory. We refer the reader to $[9,10]$ for more discussion on the possible applications of our methods to these research areas. Here and below we will always assume $n \geq 4$, so the smallest Springer variety we consider corresponds to the $2 \times 2$ block

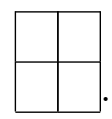

More specifically, we use the poset pinball methods introduced in [9] and the dimension pair algorithm for determining pinball rolldowns described in [2] to construct our combinatorially natural module basis for $H_{S^{1}}^{*}\left(\mathcal{S}_{(n-2,2)}\right)$. Our arguments use our results above on highest forms and the explicit correspondence between permissible fillings and $S^{1}$-fixed points of the Springer variety. The module basis is obtained by taking images under the natural projection map $H_{\mathbb{T}}^{*}\left(\mathcal{F}\right.$ lags $\left.\left(\mathbb{C}^{n}\right)\right) \rightarrow H_{S^{1}}^{*}\left(\mathcal{S}_{(n-2,2)}\right)$, to be described in detail below, of a subset of the $\mathbb{T}$-equivariant Schubert classes in $H_{\mathbb{T}}^{*}\left(\mathcal{F}\right.$ lags $\left.\left(\mathbb{C}^{n}\right)\right)$. A similar analysis by Bayegan and the second author in a special case of regular nilpotent Hessenberg varieties [2] yields a poset-upper-triangular basis in the sense of [9]. In contrast to the results in [2], in the present manuscript we find that the module basis is not poset-uppertriangular; this is the first such example in the literature. In addition, a straightforward consequence of our proof is that a simple change of variables yields a module basis which is not a poset pinball basis but is poset-upper-triangular. These results provide further evidence for the point of view, explained in [9], that geometrically natural GKM-type module bases can be computationally convenient even if not poset-upper-triangular.

We now outline the contents of the paper. In Section 2 we provide the necessary definitions and set some notation. In Section 3 we define the adjacent-pair matrix and prove results concerning highest forms of linear operators. As a simple application we derive the change-of-variable matrix required to describe the circle subgroup of $\mathbb{T} \subseteq U(n, \mathbb{C})$ acting on a translated Springer variety. Section 4 contains our results on the bijection between permissible fillings of a Young diagram and the $S^{1}$-fixed points of Hessenberg varieties. Section 5 is a mainly expository section which recalls the terminology and definitions of poset pinball and the dimension pair algorithm in $[2,9]$. In Sections 6 and 7, poset pinball for the case of $(n-2,2)$ Springer varieties is studied in detail. The small- $n$ cases $n=4$ and $n=5$ are explicitly computed and recorded in Section 6 . The main pinball result is in Section 7, where we prove that the dimension pair algorithm yields a linearly independent set of classes in $H_{S^{1}}^{*}\left(\mathcal{S}_{(n-2,2)}\right)$ and hence a module basis. We close with some directions for future investigation in Section 8.

Acknowledgements. We thank Darius Bayegan, Erik Insko, and Aba Mbirika for helpful conversations and interest in this project. We are particularly grateful to Julianna Tymoczko for her ongoing support, as well as for finding (and suggesting ways to fix!) errors in an earlier draft of this paper and making many excellent suggestions for improving exposition. We also thank the anonymous referee for a careful reading of our manuscript and numerous helpful comments. 


\section{Nilpotent Hessenberg varieties and $S^{1}$-actions}

We begin with the definition of the type $A$ nilpotent Hessenberg varieties, of which the nilpotent Springer varieties are a special case. We also recall the definition of a circle subgroup of the maximal torus $\mathbb{T}$ of $U(n, \mathbb{C})$ which acts on any nilpotent Hessenberg variety. Since some of the discussion below applies to any nilpotent Hessenberg variety, we present the general definition here. We work exclusively with type $A$ in this manuscript and hence omit it from our terminology below.

Given a nilpotent operator $N: \mathbb{C}^{n} \rightarrow \mathbb{C}^{n}$, consider its Jordan canonical form with weakly decreasing sizes of Jordan blocks. Let $\lambda_{N}$ denote the partition of $n$ with entries the sizes of the Jordan blocks of $N$. Throughout this manuscript we identify partitions of $n$ with the corresponding Young diagram. For example, if $N: \mathbb{C}^{5} \rightarrow \mathbb{C}^{5}$ is the operator with corresponding matrix

$$
\left[\begin{array}{lllll}
0 & 1 & 0 & 0 & 0 \\
0 & 0 & 1 & 0 & 0 \\
0 & 0 & 0 & 0 & 0 \\
0 & 0 & 0 & 0 & 1 \\
0 & 0 & 0 & 0 & 0
\end{array}\right]
$$

with respect to the standard basis of $\mathbb{C}^{5}$, then since the matrix has 2 Jordan blocks of sizes 3 and 2 respectively, it has associated Young diagram

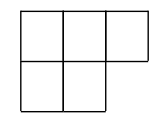

which in turn corresponds to the partition $\lambda_{N}=(3,2)$.

A Hessenberg function is a function $h:\{1,2, \ldots, n\} \rightarrow\{1,2, \ldots, n\}$ satisfying $h(i) \geq i$ for all $1 \leq i \leq n$ and $h(i+1) \geq h(i)$ for all $1 \leq i<n$. We frequently denote a Hessenberg function by listing its values in sequence $h=(h(1), h(2), \ldots, h(n)=n)$.

The (nilpotent) Hessenberg variety $\operatorname{Hess}(N, h)$ associated to $N$ and a Hessenberg function $h$ is a subvariety of the flag variety $\mathcal{F l a g s}\left(\mathbb{C}^{n}\right) \cong G L(n, \mathbb{C}) / B$. Recall that $\mathcal{F}$ lags $\left(\mathbb{C}^{n}\right)$ is the projective variety of nested subspaces in $\mathbb{C}^{n}$, i.e.

$\mathcal{F}$ lags $\left(\mathbb{C}^{n}\right)=\left\{V_{\bullet}=\left(V_{i}\right): 0 \subseteq V_{1} \subseteq V_{2} \subseteq \cdots \subseteq V_{n-1} \subseteq V_{n}=\mathbb{C}^{n}\right.$ such that $\left.\operatorname{dim}_{\mathbb{C}}\left(V_{i}\right)=i\right\}$

Then $\operatorname{Hess}(N, h)$ is defined to be the following subvariety of $\mathcal{F}$ lags $\left(\mathbb{C}^{n}\right)$ :

$$
\operatorname{Hess}(N, h):=\left\{V_{\bullet} \in \mathcal{F l a g s}\left(\mathbb{C}^{n}\right) \mid N V_{i} \subseteq V_{h(i)} \text { for all } 1 \leq i \leq n\right\}
$$

The (nilpotent) Springer varieties ${ }^{3}$ are Hessenberg varieties for the special case where the Hessenberg function is the identity function $h(i)=i$ for all $1 \leq i \leq n$ :

\footnotetext{
${ }^{3}$ In the literature they are also called Springer fibres because they arise as fibres of the symplectic resolution $T^{*} \mathcal{F} \operatorname{lags}\left(\mathbb{C}^{n}\right) \rightarrow \mathcal{N}$ where $\mathcal{N}$ denotes the subspace of nilpotent matrices in $\mathfrak{g l}(n, \mathbb{C})$, but we do not need or use this perspective here.
} 
Definition 2.1. Let $N: \mathbb{C}^{n} \rightarrow \mathbb{C}^{n}$ be a nilpotent operator. The Springer variety $\mathcal{S}_{N}$ associated to $N$ is defined as

$$
\mathcal{S}_{N}:=\left\{V_{\bullet} \in \mathcal{F} \text { lags }\left(\mathbb{C}^{n}\right) \mid N V_{i} \subseteq V_{i} \text { for all } 1 \leq i \leq n\right\}
$$

For any $g \in G L(n, \mathbb{C})$, it is straightforward to see that the Hessenberg variety $\operatorname{Hess}\left(g N g^{-1}, h\right)$ for the conjugate $g N g^{-1}$ of $N$ is homeomorphic (in fact, isomorphic as algebraic varieties) to $\operatorname{Hess}(N, h)$, with explicit homeomorphism given by translation by $g$, i.e.,

$$
\begin{gathered}
\operatorname{Hess}(N, h) \longrightarrow \operatorname{Hess}\left(g N g^{-1}, h\right) \\
k \longmapsto g k
\end{gathered}
$$

where $k \in G L(n, \mathbb{C})$ denotes a flag $[k] \in G L(n, \mathbb{C}) / B \cong \mathcal{F}$ lags $\left(\mathbb{C}^{n}\right)$.

There exists a circle action on any nilpotent Hessenberg variety. Recall first that the maximal torus $\mathbb{T}$ of $U(n, \mathbb{C})$, identified with the diagonal subgroup of $U(n, \mathbb{C})$, acts on the flag variety $\mathcal{F}$ lags $\left(\mathbb{C}^{n}\right)$. Consider the following circle subgroup of $\mathbb{T}$ :

$$
S^{1}:=\left\{\left[\begin{array}{cccc}
t^{n} & 0 & \cdots & 0 \\
0 & t^{n-1} & & 0 \\
0 & 0 & \ddots & 0 \\
0 & 0 & & t
\end{array}\right] \mid t \in \mathbb{C},\|t\|=1\right\} \subseteq \mathbb{T} \subseteq U(n, \mathbb{C}) .
$$

It is shown in [9, Lemma 5.1] that the $S^{1}$ of (3) preserves the nilpotent Hessenberg variety $\operatorname{Hess}(N, h) \subseteq \mathcal{F}$ lags $\left(\mathbb{C}^{n}\right)$ when the nilpotent operator $N$ has matrix in Jordan canonical form with respect to the standard basis of $\mathbb{C}^{n}$. Moreover, the $S^{1}$-fixed points $\operatorname{Hess}(N, h)^{S^{1}}$ are isolated and are a subset of $\mathcal{F}$ lags $\left(\mathbb{C}^{n}\right)^{\mathbb{T}}$, the $\mathbb{T}$-fixed points of $\mathcal{F}$ lags $\left(\mathbb{C}^{n}\right)$. Using the identification $\mathcal{F}$ lags $\left(\mathbb{C}^{n}\right)^{\mathbb{T}} \cong S_{n}$ we henceforth think of $S^{1}$-fixed points of $\operatorname{Hess}(N, h)$ as permutations in $S_{n}$.

\section{Adjacent-pair matrices and highest forms of nilpo- tent operators}

Let $N_{0}$ be a nilpotent matrix in Jordan canonical form with weakly decreasing Jordan block sizes. We think of $N_{0}$ as a linear operator on $\mathbb{C}^{n}$ written with respect to the standard basis of $\mathbb{C}^{n}$. As mentioned in Section 2, in addition to the Hessenberg variety $\operatorname{Hess}\left(N_{0}, h\right)$ we may also consider the translated Hessenberg varieties $\operatorname{Hess}\left(g N_{0} g^{-1}, h\right)=g \cdot \operatorname{Hess}\left(N_{0}, h\right)$ for various $g \in G L(n, \mathbb{C})$. For the purposes of poset pinball (discussed in more detail in Section 5) it turns out to be necessary to use conjugates $\sigma N \sigma^{-1}$ where $\sigma$ is a permutation matrix and $\sigma N \sigma^{-1}$ is in a so-called highest form [17, Definition 4.2]; this is because Tymoczko's construction of a paving-by-affines of a $\operatorname{Hessenberg}$ variety $\operatorname{Hess}(N, h)[17$, Theorem 6.1] assumes that $N$ is in a highest form. Motivated by this, in this section we develop a theory which relates highest forms of $N_{0}$ with fillings of the corresponding 
Young diagram $\lambda=\lambda_{N_{0}}$. First we introduce a bijection $\phi_{\lambda}: \mathcal{F} i \ell \ell(\lambda) \rightarrow S_{n}$ from the set of fillings $\mathcal{F} i \ell \ell(\lambda)$ of $\lambda$ to the permutation group $S_{n}$. Secondly we associate to each filling $T$ of $\lambda$ a matrix $N_{T}$ which we call the adjacent-pair matrix of $T$. The main result of this section is Theorem 3.21, in which we prove that $N_{T}=\sigma N_{0} \sigma^{-1}$ is in a highest form precisely when $T$ arises from a certain simple algorithm which we describe below. This yields a straightforward enumeration of all permutation matrices $\sigma$ for which $\sigma N_{0} \sigma^{-1}$ is in a highest form, and in particular in Corollary 3.26 we give a count of the number of conjugates $\sigma N_{0} \sigma^{-1}$ for $\sigma \in S_{n}$ which are in a highest form.

The discussion in this section has several motivations and consequences. Firstly, our results (e.g. Corollary 3.23) both make explicit and also generalize a procedure for producing highest forms of linear operators which is sketched in $[17$, Section 4, text near Figure 4]. Secondly, our explicit correspondence between certain fillings of $\lambda$ and highest forms of $N_{0}$ allows us to easily determine the permutation $\sigma=\phi_{\lambda}(T)$ (see e.g. Example 3.27) and thus make further explicit computations with $\sigma$. As a sample computation and for use in Section 7, at the end of this section we give a concrete description in coordinates of the conjugated circle $\sigma S^{1} \sigma^{-1}$ which acts on the Springer variety $\mathcal{S}_{\sigma N_{0} \sigma^{-1}}$ for $N_{0}$ corresponding to $\lambda=(n-2,2)$, as well as a computation of the associated Lie algebra projection $\operatorname{Lie}(\mathbb{T}) \rightarrow \operatorname{Lie}\left(\sigma S^{1} \sigma^{-1}\right)$. Thus some of the results in this section are preliminary to the arguments in the sections below. Third, we believe that the theory of highest forms in relation to poset pinball and Springer varieties will be useful in the future; we describe some open questions motivated by this in Section 8 .

We recall some definitions.

Definition 3.1. ( $[\mathbf{1 7}$, Definition 4.1]) Let $X$ be any $m \times n$ matrix. We call the entry $X_{i k}$ a pivot of $X$ if $X_{i k}$ is nonzero and if all entries below and to its left vanish, i.e., $X_{i j}=0$ if $j<k$ and $X_{j k}=0$ if $j>i$.

Moreover, given $i$, define $r_{i}$ to be the row of $X_{r_{i}, i}$ if the entry is a pivot, and 0 otherwise.

Example 3.2. Let

$$
X=\left[\begin{array}{llll}
0 & 1 & 1 & 0 \\
0 & 0 & 5 & 0 \\
0 & 1 & 0 & 0 \\
0 & 0 & 0 & 3
\end{array}\right] .
$$

Then $r_{1}=0, r_{2}=3, r_{3}=2$, and $r_{4}=4$.

Definition 3.3. ( $[\mathbf{1 7}$, Definition 4.2]) An upper-triangular nilpotent $n \times n$ matrix is in a highest form if its pivots form a nondecreasing sequence, namely $r_{1} \leq r_{2} \leq \cdots \leq r_{n}$.

Example 3.4. The nilpotent matrix

$$
N=\left[\begin{array}{llllll}
0 & 0 & 0 & 0 & 0 & 0 \\
0 & 0 & 0 & 1 & 0 & 0 \\
0 & 0 & 0 & 0 & 1 & 0 \\
0 & 0 & 0 & 0 & 0 & 0 \\
0 & 0 & 0 & 0 & 0 & 1 \\
0 & 0 & 0 & 0 & 0 & 0
\end{array}\right]
$$


is in a highest form since $r_{1}=r_{2}=r_{3}=0, r_{4}=2, r_{5}=3, r_{6}=5$.

Recall that a filling of $\lambda$ by the alphabet $[n]:=\{1,2, \ldots, n\}$ is an injective placing of the integers $\{1,2, \ldots, n\}$ into the boxes of $\lambda$. Following tableaux notation we denote by $T$ a filling of $\lambda$ by $[n]$. We denote by $\mathcal{F} i \ell \ell(\lambda)$ the set of all fillings of $\lambda$ by $[n]$. For $\lambda$ a Young diagram with $n$ boxes, we have $|\mathcal{F} i \ell \ell(\lambda)|=n$ !. In the theory below we use a particular bijective correspondence between $\mathcal{F} i \ell \ell(\lambda)$ and $S_{n}$. We introduce the following terminology.

Definition 3.5. Let $\lambda$ be a Young diagram. Let $T$ be a filling of $\lambda$ with alphabet $[n]$ for some $n \in \mathbb{N}$. By the English reading of $T$ we mean the reading of the entries of $T$ from left to right along rows, starting at the top row and proceeding in sequence to the bottom row. The word of $T$ obtained via the English reading of $T$ is called the English word of $T$. If $\lambda$ is a Young diagram with $n$ boxes then we define

$$
\phi_{\lambda}: \mathcal{F} i \ell \ell(\lambda) \rightarrow S_{n}
$$

where $\phi_{\lambda}(T)$ is the permutation whose one-line notation is given by the English word of $T$. Finally, if $\lambda$ has $n$ boxes then the English filling of $\lambda$ is the filling $T$ such that $\phi_{\lambda}(T)$ is the identity permutation in $S_{n}$.

For $\lambda$ a Young diagram with $n$ boxes, it is immediate from the definition that $\phi_{\lambda}$ is a bijection from $\mathcal{F} i \ell \ell(\lambda)$ to $S_{n}$.

Example 3.6. For

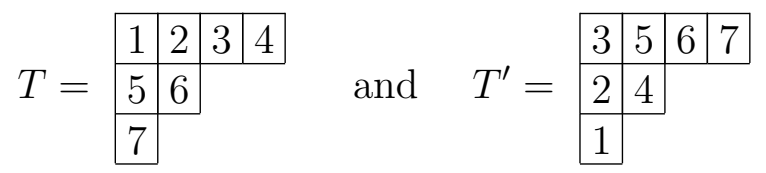

we have that $\phi_{\lambda}(T)$ and $\phi_{\lambda}\left(T^{\prime}\right)$ are respectively the permutations (in one-line notation) 1234567 and 3567241. Moreover $T$ is the English filling of $\lambda=(4,2,1)$.

Next we introduce a different reading of fillings which appears in the theory of highest forms and Hessenberg varieties developed by Tymoczko in [17] (but the terminology we use is new). In particular, this reading plays a significant role in our poset pinball methods in Sections 5-7 (cf. in particular Theorem 5.5).

Definition 3.7. Let $\lambda$ be a Young diagram. Let $T$ be a filling of a Young diagram with alphabet $[n]$ for some $n \in \mathbb{N}$. By the rotated English reading of $T$ we mean the reading of the entries of $T$ from the bottom to the top along columns, starting at the leftmost column and proceeding to the rightmost column. The word of $T$ obtained via the rotated English reading is the rotated English word of $T$. Let $\lambda$ be a Young diagram with $n$ boxes. The rotated English filling of $\lambda$ is the filling $T$ of $\lambda$ with $[n]$ such that its rotated English reading is the identity permutation in $S_{n}$. 


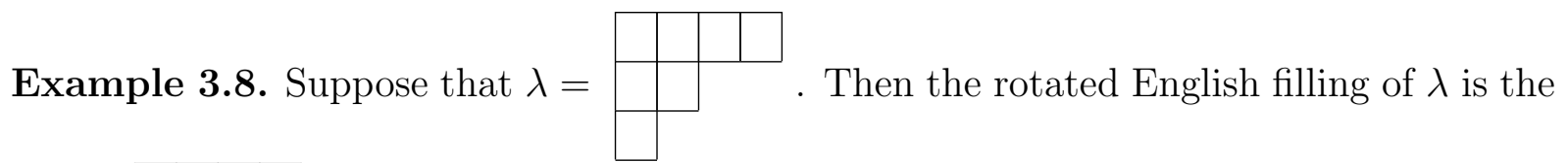
filling \begin{tabular}{|l|l|l|l|}
\hline 3 & 5 & 6 & 7 \\
\cline { 2 - 3 } 2 & 4 & \multicolumn{3}{|c}{} \\
\cline { 1 - 2 } 1 & \multicolumn{3}{|c}{} \\
&
\end{tabular}

Remark 3.9. Note that the rotated English filling is not the same thing as the conjugate of the English filling of the conjugate Young diagram. For instance for the $\lambda$ in Example 3.8 the conjugate of the English filling of the conjugate Young diagram $\tilde{\lambda}$ is \begin{tabular}{|l|l|l|l|}
\hline 1 & 4 & 6 & 7 \\
\hline 2 & 5 &
\end{tabular}

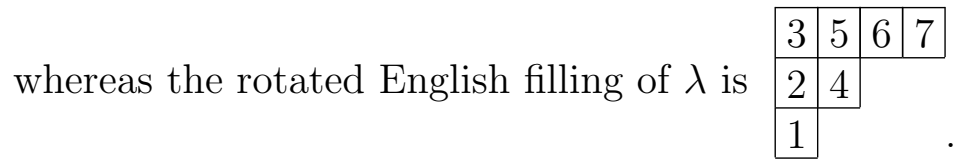

Remark 3.10. In the next section we develop a more general framework in which both Definition 3.5 and Definition 3.7 are special cases, but we do not need this perspective here.

Given a Young diagram with $n$ boxes and any filling $T$ of $\lambda$ by $[n]$, we now construct a matrix we call the adjacent-pair matrix. Our construction is a generalization of a procedure sketched by Tymoczko in [17, Section 4] (see in particular [17, Figure 4]). We begin by defining adjacency in $\lambda$ and in a filling $T$.

Definition 3.11. Let $\lambda$ be a Young diagram. We say that two boxes of $\lambda$ are adjacent if the two boxes are in the same row, and one box is directly to the left of the other. That is, the two boxes are of the form $\square$ within the Young diagram $\lambda$. Similarly, given a filling $T$ of $\lambda$, we say that two entries of $T$ are adjacent, or that they form an adjacent pair, if they occur in adjacent boxes.

Example 3.12. For

$$
T=\begin{array}{|l|l|l|}
\hline 1 & 2 & 3 \\
\hline 4 & 5 & \\
\cline { 1 - 1 } 6 & \multicolumn{2}{|l|}{} \\
\cline { 1 - 1 }
\end{array}
$$

the pairs $\{1,2\},\{2,3\}$, and $\{4,5\}$ are the adjacent pairs of entries of $T$.

Definition 3.13. Let $\lambda$ be a Young diagram with $n$ boxes and $T$ a filling of $\lambda$ with entries from $[n]$. Then we define the adjacent-pair matrix corresponding to $T$, denoted $N_{T}$, to be the matrix $N_{T}=\left(a_{i j}\right)_{1 \leq i, j \leq n}$ such that its $(i, j)$-th entry is given by

$$
a_{i j}:= \begin{cases}1 & \text { if } i \text { and } j \text { are adjacent in } T \text { and } i \text { is left of } j \\ 0 & \text { otherwise. }\end{cases}
$$




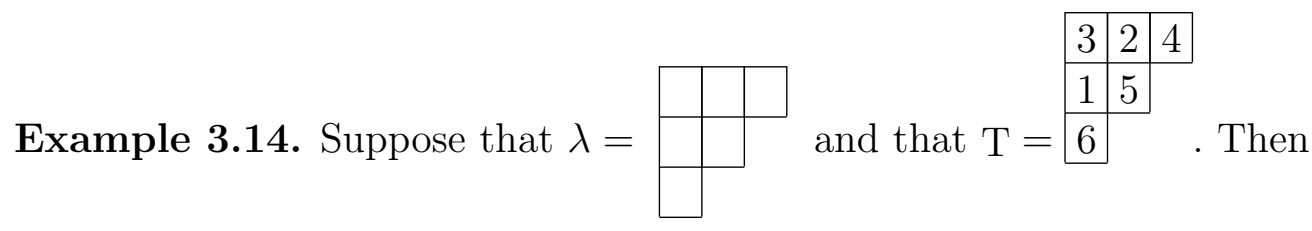

$$
N_{T}=\left[\begin{array}{llllll}
0 & 0 & 0 & 0 & 1 & 0 \\
0 & 0 & 0 & 1 & 0 & 0 \\
0 & 1 & 0 & 0 & 0 & 0 \\
0 & 0 & 0 & 0 & 0 & 0 \\
0 & 0 & 0 & 0 & 0 & 0 \\
0 & 0 & 0 & 0 & 0 & 0
\end{array}\right] .
$$

Remark 3.15. The adjacent-pair matrix $N_{T}$ corresponding to the English filling of $\lambda$ is the nilpotent matrix in Jordan canonical form corresponding to $\lambda$. For example if $\mathrm{T}=$\begin{tabular}{|l|l|l|}
\hline 1 & 2 & 3 \\
\hline 4 & 5 & \\
\hline
\end{tabular} then

$$
N_{T}=\left[\begin{array}{llllll}
0 & 1 & 0 & 0 & 0 & 0 \\
0 & 0 & 1 & 0 & 0 & 0 \\
0 & 0 & 0 & 0 & 0 & 0 \\
0 & 0 & 0 & 0 & 1 & 0 \\
0 & 0 & 0 & 0 & 0 & 0 \\
0 & 0 & 0 & 0 & 0 & 0
\end{array}\right]
$$

The following is a basic computation which relates adjacent-pair matrices to highest forms. Although simple, requiring no more than undergraduate algebra, we find this formulation most convenient for our purposes so we record it here. Given a permutation $\sigma \in S_{n}$ by slight abuse of notation we denote also by $\sigma$ its $n \times n$ permutation matrix with respect to the standard basis of $\mathbb{C}^{n}$, i.e., the matrix with $i$-th column equal to the standard basis vector $e_{\sigma(i)}$.

Lemma 3.16. Let $N$ be an $n \times n$ nilpotent matrix in Jordan canonical form with weakly decreasing sizes of Jordan blocks. Let $\lambda_{N}$ be the corresponding Young diagram. Let $T$ be a filling on $\lambda_{N}$ with alphabet $[n]$ and $\sigma:=\phi_{\lambda}(T) \in S_{n}$ the permutation given by the English word of $T$. Then the adjacent-pair matrix $N_{T}$ corresponding to $T$ is equal to the conjugate $\sigma N \sigma^{-1}$.

Proof. Both $N_{T}$ and $\sigma N \sigma^{-1}$ are matrices which contain a 1 in precisely $n-\ell$ entries and 0's elsewhere. Hence, it suffices to check that the $(i, j)$-th entry of $N_{T}$ is a 1 if and only if the same holds for $\sigma N \sigma^{-1}$. This can be easily checked using the definition of the adjacent-pair matrix and the English reading.

Remark 3.17. Observe, from Lemma 3.16, that an adjacent-pair matrix is a permutation matrix with some entries changed to 0. 
We now wish to determine the set of fillings $T$ such that the adjacent-pair matrix $N_{T}$ is in a highest form. Throughout this discussion we use the following assumptions and notation. Let $\lambda$ be a Young diagram with $n$ boxes, $\ell$ rows, $k$ rows of distinct length, and $r$ columns. If $\lambda_{1}>\lambda_{2}>\cdots>\lambda_{k}$ are the distinct row lengths of $\lambda$ we let $d_{i}$ for $1 \leq i \leq k$ denote the number of rows of $\lambda$ with length $\lambda_{i}$. Thus the row lengths of $\lambda$ are

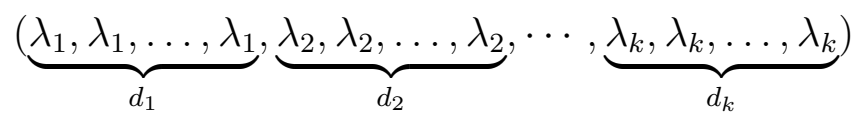

with $\sum_{i=1}^{k} d_{i}=\ell$. We also let $\left(\mu_{1} \geq \mu_{2} \geq \cdots \geq \mu_{r}\right)$ denote the column lengths of $\lambda$. Note $\mu_{1}=\ell$.

We begin with some observations about the pivots of an adjacent-pair matrix $N_{T}$.

Lemma 3.18. Let $\lambda$ be a Young diagram with $n$ boxes and $T$ a filling of $\lambda$ by $[n]$. Let $N_{T}$ be the adjacent-pair matrix of $T$. Then $N_{T}$ is in a highest form if and only if $T$ satisfies the following conditions:

(a) the leftmost column of $\lambda$ is filled with the integers $\left\{1,2, \ldots, \mu_{1}=\ell\right\}$, and

(b) if \begin{tabular}{|l|l|}
$i_{1}$ & $j_{1}$ \\
\hline
\end{tabular} and \begin{tabular}{l|l|l|}
\hline$i_{2}$ & $j_{2}$ \\
\hline
\end{tabular}

$$
i_{1}<i_{2} \text { if and only if } j_{1}<j_{2} \text {. }
$$

Proof. First suppose $N_{T}$ is in a highest form. By Remark 3.17, every nonzero entry in

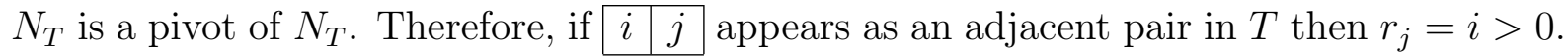
For $j \in[n]$, the index $j$ does not appear in the right hand box of any adjacent pair in $T$ (so the $j$-th column of $N_{T}$ is identically 0 ) precisely when $j$ appears in the leftmost (i.e. first) column of $\lambda$. In this case, by definition of pivots, $r_{j}=0$. Since $N_{T}$ is in a highest form we must have $r_{1} \leq r_{2} \leq \cdots \leq r_{n}$ and in particular any $r_{j}$ with $r_{j}=0$ must occur before any $r_{j}$ with $r_{j}>0$. We conclude that $j$ is in the leftmost column of $\lambda$

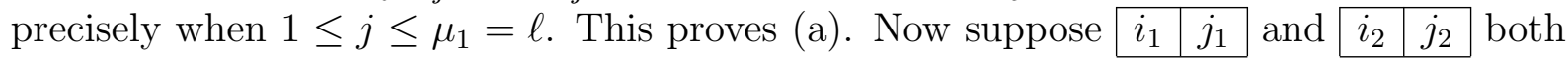
appear as adjacent pairs in $T$. Then again since all nonzero entries are pivots, we know $r_{j_{1}}=i_{1}, r_{j_{2}}=i_{2}$. If $N_{T}$ is in a highest form then the pivots must be increasing so $j_{1}<j_{2}$ if and only if $i_{1}<i_{2}$. This proves (b). If $T$ satisfies conditions (a) and (b) then reversing this reasoning shows that $N_{T}$ must be in a highest form.

We now describe an algorithm which produces a filling $T$ of $\lambda$ which satisfies certain conditions, starting from the data of a filling of the leftmost column of $\lambda$. As we show in Theorem 3.21 below, the algorithm gives an explicit method for producing precisely those fillings $T$ for which the corresponding $N_{T}$ are in a highest form. We follow notation established above.

1. Fix an arbitrary filling of the leftmost (i.e. first) column of $\lambda$ with the alphabet $\left[\mu_{1}\right]$. This filling specifies a linear ordering of the rows of $\lambda$.

2. For the $s$-th column of $\lambda$ for $2 \leq s \leq r$, place the $\mu_{s}$ integers $\left\{\left(\sum_{t=1}^{s-1} \mu_{t}\right)+1, \ldots, \sum_{t=1}^{s} \mu_{t}\right\}$ in the $\mu_{s}$ boxes of the $s$-th column in the linear order specified by step (1). 
Note that, by definition of this algorithm, the filling of the leftmost column completely specifies the rest of the filling.

Example 3.19. If the Young diagram $\lambda$ and the initial filling of its leftmost column are
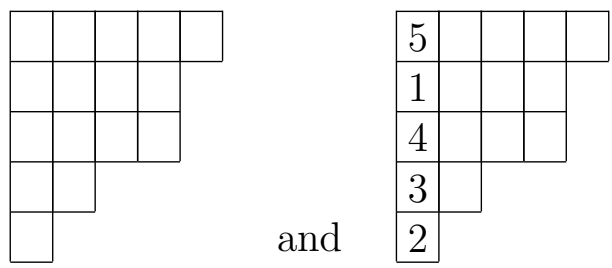

then the algorithm (5) determines the rest of the filling to be

\begin{tabular}{|c|c|c|c|}
\hline $5 !$ & \begin{tabular}{l|l}
9 & 12
\end{tabular} & 15 & 16 \\
\hline 10 & \begin{tabular}{l|l}
6 & 10
\end{tabular} & 13 & \\
\hline 48 & \begin{tabular}{l|l}
8 & 11 \\
\end{tabular} & 14 & \\
\hline $\begin{array}{l}37 \\
\end{array}$ & 7 & & \\
\hline 2 & & & \\
\hline
\end{tabular}

Remark 3.20. Suppose the filling of the leftmost column of $\lambda$ is given by placing the integer $i$, for $1 \leq i \leq \mu_{1}$, in the $i$-th box from the bottom. Then the filling of $\lambda$ obtained by applying the algorithm (5) is precisely the rotated English filling of Definition 3.7.

We now prove that the fillings $T$ for which $N_{T}$ is in a highest form are precisely those produced from the algorithm (5).

Theorem 3.21. Let $\lambda$ be a Young diagram with $n$ boxes and $T$ a filling of $\lambda$ by $[n]$. Then the adjacent-pair matrix $N_{T}$ is in a highest form if and only if the algorithm (5) applied to the filling of the leftmost column of $T$ produces the filling $T$.

To prove the proposition we use the following lemma. We use the notation established above.

Lemma 3.22. Let $\lambda$ be a Young diagram with $n$ boxes and $T$ a filling of $\lambda$ by $[n]$. Suppose $T$ satisfies the conditions (a) and (b) of Lemma 3.18. Then the $s$-th column of $\lambda$ for $1 \leq s \leq r$ contains precisely the integers $\left\{\left(\sum_{t=1}^{s-1} \mu_{t}\right)+1, \ldots, \sum_{t=1}^{s} \mu_{t}\right\}$.

Proof. We sketch an argument by induction. Condition (a) already implies the leftmost column is filled with $\left[\mu_{1}\right]$, which proves the base case $s=1$. Assume the conclusion holds for the first $s$ columns, and suppose for a contradiction that it fails for the $(s+1)$ th column. It is then straightforward to construct two adjacent pairs violating condition (b). The result follows.

Proof of Theorem 3.21. By Lemma 3.18 it suffices to prove that a filling $T$ satisfies conditions (a) and (b) of Lemma 3.18 if and only if it arises from (5). So suppose $T$ satisfies Lemma 3.18(a) and (b). From Lemma 3.22 we already know that the set of entries in each column agrees with that specified by (5), so it remains to show that the ordering 
of the entries also agrees, i.e. that the entries of the $s$-th column for $2 \leq s \leq r$ respects the linear order imposed on the rows by the filling of the leftmost column. We argue by induction. Suppose $s=2$. Then the entries of the 2 nd column respect the ordering in the 1st column precisely when the following holds: if $i_{1} j_{1}$ and \begin{tabular}{ll|l|}
$i_{2}$ & $j_{2}$
\end{tabular} are two adjacent pairs with $j_{1}, j_{2}$ in the 2 nd column of $\lambda$ then $i_{1}<i_{2}$ if and only if $j_{1}<j_{2}$. But this follows from condition (b). Moreover if this condition holds it follows that the linear ordering of the boxes in the 2 nd column given by its filling by $\left\{\mu_{1}+1, \ldots, \mu_{1}+\mu_{2}\right\}$ agrees with that induced by the linear ordering of the rows of $\lambda$ corresponding to the filling of the 1st column. Assuming the first $s$ columns are obtained by (5), the same argument as above shows that the $(s+1)$ st column must also be filled according to $(5)$, as desired.

Conversely, suppose $T$ is obtained from (5). By construction $T$ satisfies condition (a). Now suppose \begin{tabular}{|l|l|}
$i_{1}$ & $j_{1}$ \\
\hline
\end{tabular} and \begin{tabular}{|l|l|l}
$i_{2}$ & $j_{2}$ \\
\hline
\end{tabular} are two adjacent pairs appearing in $T$. We consider cases. Suppose $i_{1}$ and $i_{2}$ appear in the $s$ th and $s^{\prime}$ th columns of $T$. Without loss of generality we may assume $s<s^{\prime}$. Then $i_{1} \leq \sum_{t=1}^{s} \mu_{t}$ while $i_{2} \geq\left(\sum_{t=1}^{s^{\prime}-1} \mu_{t}\right)+1 \geq i_{1}$. Thus we wish to show $j_{1}<j_{2}$. This follows because the adjacency with $i_{1}$ and $i_{2}$ respectively implies that $j_{1}$ is in the $(s+1)$ th column and $j_{2}$ is in the $\left(s^{\prime}+1\right)$ th column. Since $s+1<s^{\prime}+1$ an argument similar to that above implies $j_{1}<j_{2}$ as desired. On the other hand suppose $i_{1}$ and $i_{2}$ appear in the same column, say the sth. Then $j_{1}$ and $j_{2}$ appear in the $s+1$ th column. Suppose further that $i_{1}$ appears in the $r_{i_{1}}$ th row and $i_{2}$ appears in the $r_{i_{2}}$ th row. If $i_{1}<i_{2}$ then by definition of the algorithm (5) the entry in the $r_{i_{1}}$ th row of the first column is less than that in the $r_{i_{2}}$ th row, which in turn implies $j_{1}<j_{2}$. Similarly $j_{1}<j_{2}$ implies $i_{1}<i_{2}$. This concludes the proof.

The following, asserted in [17, Section 4, see e.g. Figure 4], is now a straightforward consequence.

Corollary 3.23. Let $\lambda$ be a Young diagram with $n$ boxes and $T_{R E}$ be the rotated English filling of $\lambda$. Let $\sigma:=\phi_{\lambda}\left(T_{R E}\right)$ be the permutation given by the English reading of $T_{R E}$. Then $N_{T_{R E}}=\sigma N \sigma^{-1}$ is in a highest form.

Proof. Immediate from Lemma 3.16 and Theorem 3.21 and Remark 3.20.

We have just seen that each filling $T$ obtained from (5) yields a conjugate $N_{T}=\sigma N \sigma^{-1}$ of $N$ in a highest form. Since a filling given in (5) is specified by the filling of its leftmost column, there are $\mu_{1} !=\left|S_{\mu_{1}}\right|$ many such fillings. However, different such fillings $T$ and $T^{\prime}$ may yield the same adjacent-pair matrix $N_{T}=N_{T^{\prime}}$. The next lemma makes this precise, for the purpose of which we use the following terminology. We say a filling $T^{\prime}$ is obtained from $T$ by a row swap if the entries of 2 equal-length rows of $T$ have been interchanged.

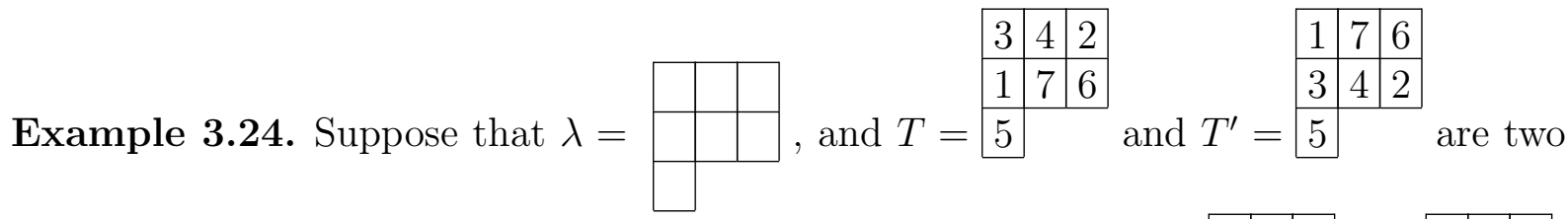

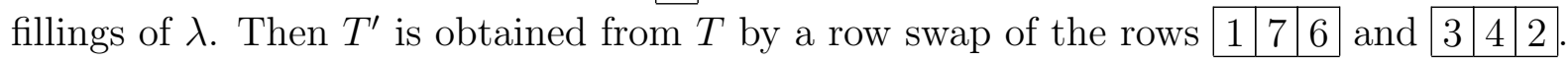


With the terminology in place, we have the following lemma.

Lemma 3.25. Let $\lambda$ be a Young diagram with $n$ boxes and let $T$ and $T^{\prime}$ be fillings of $\lambda$ obtained from (5). Then $N_{T}=N_{T^{\prime}}$ if and only if $T^{\prime}$ is obtained from $T$ by a sequence of row swaps.

Proof. If $T$ and $T^{\prime}$ differ only by a sequence of row swaps, then $T$ and $T^{\prime}$ have precisely the same sets of adjacent pairs. Thus from the definition of the adjacent-pair matrix it follows that $N_{T}=N_{T^{\prime}}$. Now suppose $T$ and $T^{\prime}$ differ by more than a sequence of row swaps. Since both $T$ and $T^{\prime}$ are obtained from (5), this means that there exists an element $s \in\left[\mu_{1}\right]$ which appears in $T$ in a row of length $d$ and appears in $T^{\prime}$ in a row of length $d^{\prime}$, with $d \neq d^{\prime}$. Without loss of generality we assume $d^{\prime}>d$. We wish to show that $N_{T} \neq N_{T^{\prime}}$. For this it suffices to show that there exists some adjacent pair $i \quad j$ which occurs in $T$ but not in $T^{\prime}$, or vice versa. Consider the entries in the row of $T$ and $T^{\prime}$ which contain $s$. By assumption these are of the form \begin{tabular}{|l|l|l|l|}
\hline$a_{1}=s$ & $a_{2}$ & $\cdots$ & $a_{d}$ \\
and
\end{tabular}

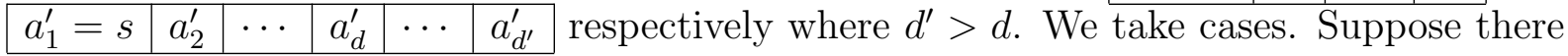
exists an index $1<i \leq d$ for which $a_{i} \neq a_{i}^{\prime}$. Then in particular there exists a minimal such, denote it $i$. Then there is an adjacent pair \begin{tabular}{|l|l|l|l|}
$a_{i-1}$ & $a_{i}$ \\
in $T$ and a pair & $a_{i-1}^{\prime}$ & $a_{i}^{\prime}$ & in
\end{tabular} $T^{\prime}$ where $a_{i-1}=a_{i-1}^{\prime}$ but $a_{i} \neq a_{i}^{\prime}$, so $N_{T} \neq N_{T^{\prime}}$. Now suppose $a_{i}=a_{i}^{\prime}$ for all $1 \leq i \leq d$. In

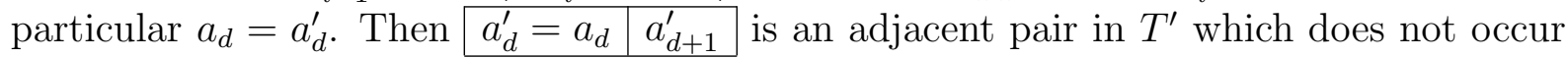
in $T$. Hence $N_{T} \neq N_{T^{\prime}}$ also in this case. The result follows.

The following is now straightforward. Recall $\mu_{1}=\ell$ is the total number of rows of $\lambda$ and $d_{1}, \ldots, d_{k}$ are the numbers of rows of $\lambda$ of length $\lambda_{1}, \ldots, \lambda_{k}$ respectively.

Corollary 3.26. There exist precisely

$$
\frac{\ell !}{d_{1} ! d_{2} ! \cdots d_{k} !}
$$

highest forms of $N$ obtained as $\sigma N \sigma^{-1}$ for a permutation matrix $\sigma \in S_{n}$.

Proof. There are $\mu_{1} !=\ell$ ! fillings $T$ arising from the algorithm (5). From Lemma 3.25 we know that the matrices $N_{T}$ do not change precisely when the entries in the first column contained in equal-length rows are permuted. The $d_{i}$ count the numbers of equal-length rows so the result follows.

Our constructions allow us to do explicit computations. For instance, given the discussion above it is straightforward to list the permutation matrices $\sigma$ for which the associated conjugate $\sigma N \sigma^{-1}$ is in a highest form. For instance, let $T_{R E}$ be the rotated English filling of $\lambda$. It follows from the results above that the permutation $\sigma$ for which $\sigma N \sigma^{-1}$ is the highest form of $N$ used in [17, Section 4] is precisely $\sigma:=\phi_{\lambda}(T)$.

Example 3.27. Consider the following Young diagram

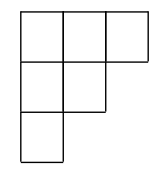


which corresponds to the nilpotent matrix $N$ in Example 3.4. Then the rotated English filling is

$$
\begin{array}{|l|l|l|}
\hline 3 & 5 & 6 \\
\hline 2 & 4 & \multicolumn{1}{|c}{} \\
\cline { 1 - 1 } 1 & \multicolumn{2}{|c}{} \\
\cline { 1 - 1 } & &
\end{array}
$$

and the permutation $\sigma$ such that $\sigma N \sigma^{-1}$ is in the highest form used in [17, Section 4$]$ is $\sigma=356241$.

As another application of our discussion and for use in Section 7 we close this section with a brief discussion about the circle action on Hessenberg varieties defined in (3). Consider the translated Hessenberg variety $\operatorname{Hess}\left(\sigma N \sigma^{-1}, h\right)$ where $N$ is in Jordan canonical form and $\sigma$ is a permutation matrix. In this case the circle subgroup of (3) does not necessarily act on Hess $\left(\sigma N \sigma^{-1}, h\right)$. Instead we consider the conjugated circle subgroup $\sigma S^{1} \sigma^{-1}$ of $\mathbb{T}$, which is easily seen to preserve $\operatorname{Hess}\left(\sigma N \sigma^{-1}, h\right)$. Here and below we consider each such Springer variety to be equipped with this conjugated circle group action, which by slight abuse of notation we sometimes denote also by $S^{1}$ (instead of $\sigma S^{1} \sigma^{-1}$ ). It is immediate that the fixed points $\operatorname{Hess}\left(\sigma N \sigma^{-1}, h\right)^{S^{1}}$ under the $S^{1}$-action are isolated and are a subset of $S_{n} \cong \mathcal{F} \operatorname{lags}\left(\mathbb{C}^{n}\right)^{\mathbb{T}}$; indeed, under the homeomorphism (2) the set of $S^{1}$-fixed points $\operatorname{Hess}\left(\sigma N \sigma^{-1}, h\right)^{S^{1}}$ is precisely the $\sigma$-translate

$$
\sigma \cdot \operatorname{Hess}(N, h)^{S^{1}} \subseteq S_{n}
$$

of the $S^{1}$-fixed points of $\operatorname{Hess}(N, h)$.

In Section 7 we focus attention on a choice of Springer variety $\mathcal{S}_{\sigma N \sigma^{-1}}$ specified by $\lambda=(n-2,2)$ with nilpotent matrix $N$ and the choice of permutation $\sigma$ determined by the rotated English filling. In this setting we give below an explicit computation of the conjugate circle subgroup $\sigma S^{1} \sigma^{-1}$ and the linear projection $\operatorname{Lie}(\mathbb{T})^{*} \rightarrow \operatorname{Lie}\left(S^{1}\right)^{*}$. We illustrate with a concrete example.

Example 3.28. Let $\lambda=(4,2)$. Then the corresponding matrix in Jordan canonical form is

$$
N=\left[\begin{array}{llllll}
0 & 1 & 0 & 0 & 0 & 0 \\
0 & 0 & 1 & 0 & 0 & 0 \\
0 & 0 & 0 & 1 & 0 & 0 \\
0 & 0 & 0 & 0 & 0 & 0 \\
0 & 0 & 0 & 0 & 0 & 1 \\
0 & 0 & 0 & 0 & 0 & 0
\end{array}\right]
$$

and the associated permutation determined from the rotated English filling is $\sigma=245613$. The standard $S^{1}$ in (3) is then conjugated to the circle subgroup

$$
S^{1} \cong \sigma S^{1} \sigma^{-1}=\left\{\left[\begin{array}{cccccc}
t^{2} & 0 & 0 & 0 & 0 & 0 \\
0 & t^{6} & 0 & 0 & 0 & 0 \\
0 & 0 & t & 0 & 0 & 0 \\
0 & 0 & 0 & t^{5} & 0 & 0 \\
0 & 0 & 0 & 0 & t^{4} & 0 \\
0 & 0 & 0 & 0 & 0 & t^{3}
\end{array}\right]\right\}
$$


The corresponding linear projection $\operatorname{Lie}\left(\mathbb{T}^{6}\right)^{*}=\mathfrak{t}^{*} \rightarrow \operatorname{Lie}\left(S^{1}\right)^{*}$ induced by the inclusion $S^{1} \cong \sigma S^{1} \sigma^{-1} \hookrightarrow \mathbb{T}^{6}$ is given by

$$
t_{1} \mapsto 2 t, \quad t_{2} \mapsto 6 t, \quad t_{3} \mapsto t, \quad t_{4} \mapsto 5 t, \quad t_{5} \mapsto 4 t, \quad t_{6} \mapsto 3 t
$$

where $t$ denotes the variable in $\operatorname{Lie}\left(S^{1}\right)$ and the $t_{i}$ the variables in $\operatorname{Lie}\left(\mathbb{T}^{6}\right) \cong \mathbb{R}^{6}$.

The general computation follows.

Lemma 3.29. Let $n \geq 4$. Let $\lambda=(n-2,2)$ and let $S^{1}$ denote the standard circle subgroup in (3). Then the permutation $\sigma$ determined by the rotated English filling of $\lambda$ is

$$
\sigma=24567 \cdots n-1 n 13
$$

in one-line notation and the conjugated subgroup $\sigma S^{1} \sigma^{-1}$ is given by

$$
S^{1} \cong \sigma S^{1} \sigma^{-1}=\left\{\left[\begin{array}{ccccccc}
t^{2} & 0 & 0 & 0 & 0 & \cdots & 0 \\
& t^{n} & & & & & \\
& & t & & & & \\
& & & t^{n-1} & & & \\
& & & t^{n-2} & & \\
& & & & & \ddots & \\
& & & & & t^{3}
\end{array}\right]\right\} \mathbb{T} .
$$

Moreover, the linear projection $\mathfrak{t}^{*} \rightarrow \operatorname{Lie}\left(S^{1}\right)^{*}$ determined by the inclusion of this circle subgroup $S^{1} \cong \sigma S^{1} \sigma^{-1} \hookrightarrow \mathbb{T}$ is given by

$$
t_{1} \mapsto 2 t, \quad t_{2} \mapsto n t, \quad t_{3} \mapsto t, \quad \text { and } t_{k} \mapsto(n+3-k) t, \text { for } 4 \leq k \leq n .
$$

Proof of Lemma 3.29. By definition $\lambda$ is the partition with $n-2$ boxes in the first row and 2 boxes in the second row. Its rotated English filling is \begin{tabular}{|l|l|l|l|l|l|l|}
\hline 2 & 4 & 5 & $\cdots$ & $n-2$ & $n-1$ & $n$ \\
\hline 1 & 3 & &
\end{tabular} from which the form of $\sigma$ (obtained by the English reading of the above filling) follows. Moreover the inverse of the given $\sigma$ is $\sigma^{-1}=n-11 n 234 \cdots n-2$. Equation (7) then follows by conjugating the circle subgroup of (3) by the $n \times n$ permutation matrix corresponding to $\sigma$. For the last statement of the lemma we identify the subgroup (7) with the standard circle $S^{1} \subseteq \mathbb{C}^{*}$ via the map $t \mapsto \operatorname{diag}\left(t^{2}, t^{n}, t, t^{n-1}, t^{n-2}, \ldots, t^{3}\right)$. The derivative of this map takes the standard basis vector in $\operatorname{Lie}\left(S^{1}\right) \cong \mathbb{R}$ to

$$
(2, n, 1, n-1, n-2, \ldots, 3)
$$

in $\mathbb{R}^{n}$ with respect to the standard basis, where the $k$-th coordinate for $4 \leq k \leq n$ is $n+3-k$. The final statement of the lemma now follows. 


\section{$4 \quad S^{1}$-fixed points in Hessenberg varieties and permis- sible fillings}

In this section we give an explicit bijection from the $S^{1}$-fixed points of $\operatorname{Hess}(N, h)$, for various choices of $N$, to the set of permissible fillings of $\lambda_{N}$. The last result of the section, Corollary 4.10, is used in Sections 5-7 but the discussion is also of independent interest. Our results further develop some ideas in [17], in which Tymoczko constructs a paving-by-affines of a nilpotent Hessenberg variety $\operatorname{Hess}(N, h)$ by using certain Schubert cells. (In [17] Tymoczko considers more general Hessenberg varieties but we focus on the nilpotent case here.) Since each Schubert cell $B w B$ in $G L(n, \mathbb{C})$ contains a unique coset $w B$ with $w$ a permutation matrix, it follows from her construction that there is a unique such $w$ associated to each of the affine cells in her paving of $\operatorname{Hess}(N, h)$, which in turn can be encoded in a filling of a Young diagram [17, Theorem 7.1]. Our main result in this section, Theorem 4.7, is another interpretation of this bijection; our main contribution is to make more explicit and precise the bijective correspondence between the permissible fillings of $\lambda_{N}$ and the cosets $w B$ for $w$ a permutation matrix which lie in $\operatorname{Hess}\left(\sigma N \sigma^{-1}, h\right)$ (thought of as $S^{1}$-fixed points of $\operatorname{Hess}\left(\sigma N \sigma^{-1}, h\right)$ ) for different choices of conjugates $\sigma N \sigma^{-1}$. We also refer the reader to [2] for related discussion; in particular, Corollary 4.10 proves a claim used in [2, Section 2].

We begin by defining permissible fillings following [13].

Definition 4.1. Let $\lambda$ be a Young diagram with $n$ boxes and $h$ a Hessenberg function. A filling of $\lambda$ is a $(h, \lambda)$-permissible filling if for every horizontal adjacency \begin{tabular}{|l|l|l|}
$k$ & we
\end{tabular} have $k \leq h(j)$. (When the $h$ and $\lambda$ are understood from context we sometimes omit the $(h, \lambda)$ from terminology and refer simply to permissible fillings.)

Remark 4.2. In the context of Springer varieties, for which $h(j)=j$ for all $j$, the condition $k \leq h(j)$ becomes $k \leq j$. Thus in this case permissible fillings are precisely the row-strict fillings.

Given $\lambda$ and $h$, we denote by

$$
\mathcal{P F i \ell \ell}(\lambda, h) \subseteq \mathcal{F} i \ell \ell(\lambda)
$$

the set of permissible fillings of $\lambda$. Let $N$ be a nilpotent $n \times n$ matrix in Jordan canonical form with corresponding Young diagram $\lambda$, and let $h:\{1,2, \ldots, n\} \rightarrow\{1,2, \ldots, n\}$ be a Hessenberg function. Our goal is to construct an explicit identification between Hessenberg fixed points $\operatorname{Hess}\left(\sigma N \sigma^{-1}, h\right)$ and permissible fillings $\mathcal{P} F i \ell \ell(\lambda, h)$ for any permutation matrix $\sigma$.

As a first step we define an identification between $S_{n}$ and $\mathcal{F} i \ell \ell(\lambda)$ which depends on the choice of permutation $\sigma$. Recall that $\phi_{\lambda}: \mathcal{F} i \ell \ell(\lambda) \rightarrow S_{n}$ is the mapping given by the English reading of a filling.

Definition 4.3. Let $\sigma$ be a permutation in $S_{n}$ and $\lambda$ a Young diagram with $n$ boxes. Consider the filling $\phi_{\lambda}^{-1}(\sigma)$ of $\lambda$ corresponding to $\sigma$ via the English reading. The filling 
$\phi_{\lambda}^{-1}(\sigma)$ specifies a linear ordering on the boxes of $\lambda$. Define the map

$$
\phi_{\lambda, \sigma}: \mathcal{F} i \ell \ell(\lambda) \rightarrow S_{n}
$$

by associating to any filling $T$ of $\lambda$ the permutation whose one-line notation is the reading of the entries of $T$ with respect to the linear ordering given by $\phi_{\lambda}^{-1}(\sigma)$.

Example 4.4. Suppose $\lambda=(3,2,1)$ and $\sigma=253416$. Then $\phi_{\lambda}^{-1}(\sigma)$ is the filling \begin{tabular}{|l|l|l|}
\hline 2 & 5 & 3 \\
\hline 4 & 1 \\
\hline 6
\end{tabular}

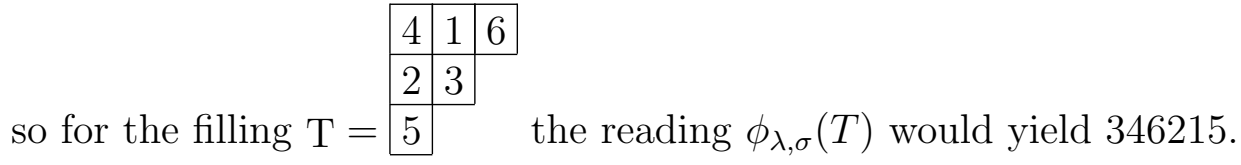

Remark 4.5. By definition the mapping $\phi_{\lambda, i d}$ corresponding to $\sigma=i d$ the identity permutation coincides with the map $\phi_{\lambda}$ obtained via the English reading. Similarly the permutation $\sigma$ for which $\phi_{\lambda, \sigma}(T)$ is the rotated English reading is precisely the permutation corresponding under $\phi_{\lambda}$ to the rotated English filling of $\lambda$.

Remark 4.5 shows that both the English and the rotated English readings of $\mathcal{F} i \ell \ell(\lambda)$ are special cases of $\phi_{\lambda, \sigma}$. The point of Definition 4.3 is to emphasize that other choices, corresponding to different choices of translated Hessenberg varieties, are possible. We need the following lemma.

Lemma 4.6. Let $\lambda$ be a Young diagram with $n$ boxes and $\sigma, \tau \in S_{n}$. Then

$$
\phi_{\lambda, \sigma}^{-1}(\tau)=\phi_{\lambda}^{-1}(\tau \sigma) .
$$

Proof. This follows from the definition of $\phi_{\lambda, \sigma}$ and the fact that multiplication by $\sigma$ on the right re-orders the entries in the one-line notation for $\tau$ precisely by replacing the $i$-th entry $\tau(i)$ by $\tau(\sigma(i))$ for all $i$.

The main theorem of this section is the following. We consider $\operatorname{Hess}\left(\sigma N \sigma^{-1}, h\right)^{S^{1}}$ to be a subset of $S_{n}$ and $\mathcal{P} F i \ell \ell(\lambda)$ to be a subset of $\mathcal{F} i \ell \ell(\lambda)$.

Theorem 4.7. Let $N$ be an $n \times n$ nilpotent matrix in Jordan canonical form with weakly decreasing sizes of Jordan blocks with respect to the standard basis of $\mathbb{C}^{n}$ and let $h:\{1,2, \ldots, n\} \rightarrow\{1,2, \ldots, n\}$ be a Hessenberg function. Let $\mathcal{P} F i \ell \ell(\lambda, h)$ denote the corresponding set of permissible fillings of $\lambda$. Let $\sigma \in S_{n}$ and denote by $\operatorname{Hess}\left(\sigma N \sigma^{-1}, h\right)$ the associated nilpotent Hessenberg variety equipped with the $S^{1}$-action described in Section 2. Then the association

$$
\Phi_{\lambda, \sigma}: w \mapsto \phi_{\lambda, \sigma}^{-1}\left(w^{-1}\right)
$$

defines a bijection from $\operatorname{Hess}\left(\sigma N \sigma^{-1}, h\right)^{S^{1}}$ to $\mathcal{P} F i \ell \ell(\lambda, h)$. 
In the proof of Theorem 4.7 we use the following terminology. Suppose $h:\{1,2, \ldots, n\} \rightarrow\{1,2, \ldots, n\}$ is a Hessenberg function. We define the Hessenberg space $H$ corresponding to $h$ to be the subspace of $\mathfrak{g l}(n, \mathbb{C})$ defined by

$$
H:=\left\{X \in \mathfrak{g l}(n, \mathbb{C}) \mid X_{i j}=0 \text { if } i>h(j)\right\}
$$

where $X_{i j}$ denotes the $(i, j)$-th entry of the matrix $X$.

Example 4.8. Suppose $h=(2,3,4,4)$. Then

$$
H=\left\{X \in \mathfrak{g l}(4, \mathbb{C}) \mid X_{3,1}=X_{4,1}=X_{4,2}=0\right\}=\left\{\left[\begin{array}{cccc}
\star & \star & \star & \star \\
\star & \star & \star & \star \\
0 & \star & \star & \star \\
0 & 0 & \star & \star
\end{array}\right]\right\} \subseteq \mathfrak{g l}(n, \mathbb{C})
$$

where the $\star$ denotes free variables.

It is straightforward to reformulate the definition (1) of Hessenberg varieties as follows: for a given Hessenberg function $h$ with corresponding Hessenberg space $H$,

$$
\operatorname{Hess}(N, h)=\left\{[g] \in G L(n, \mathbb{C}) / B \mid g^{-1} N g \in H\right\} .
$$

In particular, the $S^{1}$-fixed points of $\operatorname{Hess}(N, h)$ are precisely

$$
\operatorname{Hess}(N, h)^{S^{1}} \cong\left\{w \in S_{n} \mid w^{-1} N w \in H\right\} .
$$

We use the following lemma.

Lemma 4.9. Let $\lambda$ be a Young diagram with $n$ boxes and $h:\{1,2, \ldots, n\} \rightarrow\{1,2, \ldots, n\}$ a Hessenberg function with corresponding Hessenberg space $H$. Let $T$ be a filling of $\lambda$ by the alphabet $[n]$ and let $M$ be the $n \times n$ matrix obtained by applying the adjacency algorithm to $T$. Then

$$
T \text { is }(h, \lambda) \text {-permissible } \Longleftrightarrow M \in H .
$$

Proof. By definition of the adjacency algorithm, the $(i, j)$-th entry of $M$ is non-zero precisely when $i \quad j$ occurs in the filling of $T$. Hence by definition of $H$ the matrix $M$ is in $H$ precisely if, for all such adjacent pairs $(i, j)$ in $T$, we have $i \leq h(j)$. This is exactly the definition of a $(h, \lambda)$-permissible filling.

Proof of Theorem 4.7. We first prove the claim for the special case $\sigma=i d$. Here $\phi_{\lambda, i d}=\phi_{\lambda}$ (cf. Remark 4.5) and we want to prove the map $w \mapsto \Phi_{\lambda, i d}(w):=\phi_{\lambda}^{-1}\left(w^{-1}\right)$ defines a bijection between $\operatorname{Hess}(N, h)^{S^{1}}$ and $\mathcal{P}$ Fill $(\lambda, h)$. Since taking inverses is a bijection on $S_{n}$ and $\phi_{\lambda}$ is also a bijection from $\mathcal{F} i \ell \ell(\lambda)$ to $S_{n}$, the content of the claim is that a permutation $w$ is in $\operatorname{Hess}(N, h)^{S^{1}}$ precisely when the filling $\phi_{\lambda}^{-1}\left(w^{-1}\right)$ is permissible. Recall from (13) that

$$
w \in \operatorname{Hess}(N, h)^{S^{1}} \quad \Longleftrightarrow \quad w^{-1} N w \in H .
$$

By Lemma 3.16, the matrix $w^{-1} N w$ is precisely the adjacent-pair matrix for the filling $\phi_{\lambda}^{-1}\left(w^{-1}\right)$. The claim now follows from Lemma 4.9. The statement for arbitrary $\sigma \in S_{n}$ follows from the special case $\sigma=i d$ by Lemma 4.6 and by the equality $\operatorname{Hess}\left(\sigma N \sigma^{-1}, h\right)^{S^{1}}=\sigma \cdot \operatorname{Hess}(N, h)^{S^{1}}$. 
The following is used below in Sections 5-7 as well as in [2]. Given a Young diagram $\lambda$ with $n$ boxes, denote by $T_{R E}$ the rotated English filling of $\lambda$.

Corollary 4.10. Let $N$ be an $n \times n$ nilpotent matrix in Jordan canonical form and weakly decreasing sizes of Jordan blocks with respect to the standard basis of $\mathbb{C}^{n}$ and let $h:\{1,2, \ldots, n\} \rightarrow\{1,2, \ldots, n\}$ be a Hessenberg function. Let $\mathcal{P} F i \ell \ell(\lambda, h)$ denote the corresponding set of permissible fillings of $\lambda$. Let $\sigma=\phi_{\lambda}\left(T_{R E}\right)$ be the permutation corresponding to the rotated English filling of $\lambda$. Then

$$
\Phi_{\lambda, \sigma}: w \mapsto \phi_{\lambda, \sigma}^{-1}\left(w^{-1}\right)
$$

is a bijection from $\operatorname{Hess}\left(\sigma N \sigma^{-1}, h\right)^{S^{1}}$ to $\mathcal{P} F i \ell \ell(\lambda, h)$.

\section{Betti-acceptable pinball and linear independence}

For the rest of the manuscript we restrict attention to nilpotent Springer varieties, i.e., the case in which the Hessenberg function is the identity function $h(i)=i$ for all $1 \leq i \leq n$. In this section we recount for the convenience of the reader several ideas developed in $[2,9]$ which both motivate and are used in the next two sections in our study of a special class of nilpotent Springer varieties. First we recall the dimension pair algorithm

introduced in [2] which associates to each $S^{1}$-fixed point in a nilpotent Hessenberg variety a permutation in $S_{n}$. We also recall the interpretation of the algorithm in terms of the poset pinball game introduced in [9]. More specifically, in the case of nilpotent Springer varieties, the algorithm has an interpretation as producing the output of a successful game of Betti poset pinball, as is shown in [2, Proposition 3.6]. We keep exposition brief and refer the reader to [2] for details.

We begin with the definition of dimension pairs for the special case of the identity Hessenberg function $h(i)=i$.

Definition 5.1. Let $\lambda$ a Young diagram with $n$ boxes and $T$ a permissible filling of $\lambda$. The pair $(a, b)$ is a dimension pair of $T$ if the following conditions hold:

1. $b>a$,

2. $b$ is either

- below $a$ and in the same column, or

- anywhere in a column strictly to the left of the column of $a$,

and

3. if there exists a box with filling $c$ directly adjacent to the right of $a$, then $b \leq c$.

For a dimension pair $(a, b)$ of $T$, we will refer to $b$ as the top part of the dimension pair. 
Example 5.2. Let $\lambda=(2,2)$. For the permissible filling

$$
\begin{array}{|l|l|}
\hline 1 & 3 \\
\hline 2 & 4 \\
\hline
\end{array}
$$

the dimension pairs are $\{(1,2),(3,4)\}$.

Given a permissible filling $T$ of $\lambda$, denote by $D P^{T}$ the set of dimension pairs of $T$. For each integer $\ell$ with $2 \leq \ell \leq n$, define

$$
x_{\ell}:=\left|\left\{(a, \ell) \mid(a, \ell) \in D P^{T}\right\}\right| .
$$

We call the integral vector $\mathbf{x}=\left(x_{2}, x_{3}, \ldots, x_{n}\right)$ the list of top parts of $T$. To each such $\mathbf{x}$ we associate a permutation in $S_{n}$ as follows. As a preliminary step, for each $\ell$ with $2 \leq \ell \leq n$ define

$$
u_{\ell}(\mathbf{x}):= \begin{cases}s_{\ell-1} s_{\ell-2} \cdots s_{\ell-x_{\ell}} & \text { if } x_{\ell}>0 \\ 1 \quad \text { if } x_{\ell}=0 & \end{cases}
$$

where $s_{i}$ denotes the simple transposition $(i, i+1)$ in $S_{n}$ and 1 denotes the identity permutation. We define an association $\mathbf{x} \mapsto \omega(\mathbf{x}) \in S_{n}$ by

$$
\omega(\mathbf{x}):=u_{2}(\mathbf{x}) u_{3}(\mathbf{x}) \cdots u_{n}(\mathbf{x}) \in S_{n} .
$$

With the terminology in place we now recall the dimension pair algorithm introduced in [2]. Suppose $N$ is a nilpotent $n \times n$ matrix in Jordan canonical form and weakly decreasing sizes of Jordan blocks, with corresponding Young diagram $\lambda$. Following notation in Section 4 denote by $T_{R E}$ the rotated English filling of $\lambda$ and let $\sigma:=\phi_{\lambda}\left(T_{R E}\right)$ be the permutation such that $N_{h f}:=\sigma N \sigma^{-1}$ is the highest form of $N$ used in [17, Section 4]. The algorithm explicitly constructs, for each $S^{1}$-fixed point $w \in \mathcal{S}_{N_{h f}}^{S^{1}}$, a permutation in $S_{n}$ (which we denote $\operatorname{roll}(w)$ ).

Definition of roll $: \mathcal{S}_{N_{h f}}^{S^{1}} \rightarrow S_{n}$ :

1. Let $w \in \mathcal{S}_{N_{h f}}^{S^{1}}$ and let $\phi_{\lambda, \sigma}^{-1}\left(w^{-1}\right)$ be its corresponding permissible filling.

2. Let $D P^{\phi_{\lambda, \sigma}^{-1}\left(w^{-1}\right)}$ be the set of dimension pairs in the permissible filling $\phi_{\lambda, \sigma}^{-1}\left(w^{-1}\right)$.

3. For each $\ell$ with $2 \leq \ell \leq n$, set

$$
x_{\ell}:=\left|\left\{(a, \ell) \mid(a, \ell) \in D P^{\phi_{\lambda, \sigma}^{-1}\left(w^{-1}\right)}\right\}\right|
$$

as in $(15)$ and define $\mathbf{x}:=\left(x_{2}, \ldots, x_{n}\right)$.

4. Define $\operatorname{rol\ell }(w):=(\omega(\mathbf{x}))^{-1}$ where $\omega(\mathbf{x})$ is the permutation associated to the integer vector $\mathbf{x}$ defined in (16).

We illustrate the algorithm by an example which will be used later (cf. Section 6). 
Example 5.3. Let $\lambda=(3,2)$ and let $w=24135=s_{1} s_{3} s_{2}$. Then the associated permissible filling $\phi_{\lambda, \sigma}^{-1}\left(w^{-1}\right)$ can be computed to be

$$
\begin{array}{|l|l|l|}
\hline 1 & 2 & 5 \\
\hline 3 & 4 & \\
\hline
\end{array}
$$

and the corresponding set of dimension pairs $D P^{\phi_{\lambda, \sigma}^{-1}\left(w^{-1}\right)}$ is $\{(2,3),(2,4)\}$. (Note $(1,3)$ is not a dimension pair because $3 \nless 2$.) Here $n=5$, so the vector $\mathbf{x}$ is $(0,1,1,0)$. The permutation $\omega(\mathbf{x})$ is then 13425 , and finally $\operatorname{rol\ell }(w)=\omega(\mathbf{x})^{-1}=14235=s_{3} s_{2}$.

We call $\operatorname{roll}(w)$ the rolldown of $w$. The nomenclature is justified by the fact that the association $w \mapsto \operatorname{rol\ell }(w)$ satisfies properties which allow it to be interpreted as the rolldowns in a game of poset pinball, as introduced in [9].

Remark 5.4. The dimension pair algorithm in fact makes sense more generally, not just for $(n-2,2)$ Springer varieties. Indeed, it was originally introduced in [2] for any (type A) nilpotent Hessenberg variety. However, it is only for special cases (such as the $(n-2,2)$ Springer varieties considered here) that we are currently able to prove that the rolldowns correspond to a module basis in equivariant cohomology. It is an open question (cf. Question 8.2) whether or not the arguments in this paper can be further generalized to also yield module bases for equivariant cohomology rings of more general nilpotent Hessenberg varieties.

Our main motivation for introducing the dimension pair algorithm comes from the following geometric theorem (the statement is actually more general, but we restrict to the case at hand). The statement assumes that $N_{h f}$ is in the highest form corresponding to the rotated English filling of Definition 3.7.

Theorem 5.5. ([17, Theorem 1.1]) Let $N_{h f}: \mathbb{C}^{n} \rightarrow \mathbb{C}^{n}$ be a nilpotent matrix in the highest form chosen as above and let $\lambda:=\lambda_{N_{h f}}$. Let $\mathcal{S}_{N_{h f}}$ denote the corresponding nilpotent Springer variety. There is a paving by (complex) affine cells of $\mathcal{S}_{N_{h f}}$ such that:

- the affine cells are in one-to-one correspondence with $\mathcal{S}_{N_{h f}}^{S^{1}}$, and

- the (complex) dimension of the affine cell $C_{w}$ corresponding to a fixed point $w \in \mathcal{S}_{N_{h f}}^{S^{1}}$ is

$$
\operatorname{dim}_{\mathbb{C}}\left(C_{w}\right)=\left|D P^{\phi_{\lambda, \sigma}^{-1}\left(w^{-1}\right)}\right|
$$

where $\sigma=\phi_{\lambda}\left(T_{R E}\right)$.

For our purposes, the point of Theorem 5.5 is that, by construction of our dimension pair algorithm, the Bruhat lengths of the rolldowns of the Springer fixed points precisely encodes the Betti numbers of the Springer variety. This implies that our algorithm can in fact be viewed as a successful outcome of a game of Betti pinball, which is a refined version of poset pinball [9, Section 3.1].

One of the main goals of poset pinball, and also of this manuscript, is to build explicit module bases for equivariant cohomology rings. For this purpose, for any $u \in S_{n}$ let 
$\sigma_{u}$ denote the classical equivariant Schubert class in $H_{\mathbb{T}}^{*}\left(\mathcal{F}\right.$ lags $\left.\left(\mathbb{C}^{n}\right)\right)$ corresponding to $u$. Denote by $p_{u}$ the image of $\sigma_{u}$ under the projection

$$
\pi: H_{\mathbb{T}}^{*}\left(\mathcal{F} \operatorname{lags}\left(\mathbb{C}^{n}\right)\right) \rightarrow H_{S^{1}}^{*}\left(\mathcal{S}_{N_{h f}}\right)
$$

induced by the inclusion of groups $S^{1} \hookrightarrow \mathbb{T}$ and the $S^{1}$-equivariant inclusion of spaces $\mathcal{S}_{N_{h f}} \hookrightarrow \mathcal{F}$ lags $\left(\mathbb{C}^{n}\right)$. In analogy with the terminology in $[2,10]$, we refer to the images $p_{u}$ as Springer Schubert classes.

The goal of the remainder of this manuscript is to show that the set $\left\{p_{\text {roll }(w)}\right\}_{w \in \mathcal{S}_{N_{h f}}^{1^{1}}}$ of Springer Schubert classes, corresponding to the rolldowns of the Springer fixed points, is linearly independent over $H_{S^{1}}^{*}(\mathrm{pt})$ in $H_{S^{1}}^{*}\left(\mathcal{S}_{N_{h f}}\right)$. This achieves our goal of constructing module bases because, once linear independence is established, we may use the following

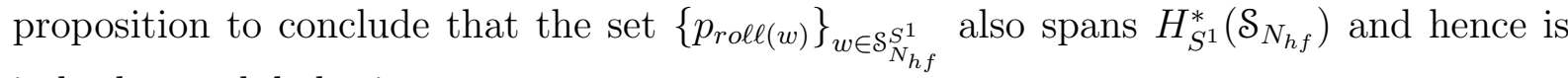
indeed a module basis.

Proposition 5.6. Let $N: \mathbb{C}^{n} \rightarrow \mathbb{C}^{n}$ be a nilpotent operator in Jordan canonical form with weakly decreasing Jordan block sizes with corresponding Young diagram $\lambda$. Let $\mathcal{S}_{N_{h f}}$ be the Springer variety corresponding to the highest form $N_{h f}:=\sigma N \sigma^{-1}$ where $\sigma$ is the permutation corresponding to the rotated English filling of $\lambda$, equipped with the $S^{1}$ action defined in (7). Let roll: $\mathcal{S}_{N_{h f}}^{S^{1}} \rightarrow S_{n}$ be the dimension-pair algorithm defined above. Suppose the classes $\left\{p_{\text {roll }(w)} \mid w \in \mathcal{S}_{N_{h f}^{1}}^{S^{1}}\right\}$ are linearly independent in $H_{S^{1}}^{*}\left(\mathcal{S}_{N_{h f}}\right)$. Then the set $\left\{p_{\text {roll }(w)} \mid w \in \mathcal{S}_{N_{h f}}^{S^{1}}\right\}$ of Springer Schubert classes form a $H_{S^{1}}^{*}(\mathrm{pt})$-module basis for the $S^{1}$-equivariant cohomology ring $H_{S^{1}}^{*}\left(\mathcal{S}_{N_{h f}}\right)$.

Proof. Since roll: $\mathcal{S}_{N_{h f}}^{S^{1}} \rightarrow S_{n}$ represents a possible outcome of a successful game of Betti poset pinball by [2, Proposition 3.7] the assertion follows from [9, Proposition 4.13].

We now briefly recall two ideas used in our proof of the linear independence of

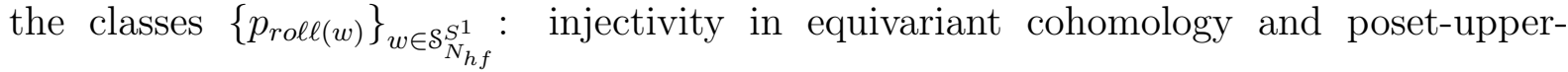
triangularity. First recall that the restriction homomorphism

$$
H_{\mathbb{T}}^{*}\left(\mathcal{F} \operatorname{lags}\left(\mathbb{C}^{n}\right)\right) \rightarrow H_{\mathbb{T}}^{*}\left(\mathcal{F} \operatorname{lags}\left(\mathbb{C}^{n}\right)^{\mathbb{T}}\right)
$$

is injective, and $\mathcal{F} \operatorname{lags}\left(\mathbb{C}^{n}\right)^{\mathbb{T}} \cong S_{n}$, so a Schubert class $\sigma_{u}$ is uniquely determined by its restrictions, denoted $\sigma_{u}(w)$, to the fixed points $w \in S_{n}$. Some known facts about the topology of Springer varieties [16] and a standard argument in equivariant cohomology (see e.g. [9, Remark 4.11 and Proposition 6.2]) shows that a similar statement holds for $H_{S^{1}}^{*}\left(\mathcal{S}_{N_{h f}}\right)$.

Proposition 5.7. Let $N: \mathbb{C}^{n} \rightarrow \mathbb{C}^{n}$ be a nilpotent operator in Jordan canonical form with weakly decreasing Jordan block sizes with corresponding Young diagram $\lambda$. Let $\mathcal{S}_{N_{h f}}$ be the Springer variety corresponding to the highest form $N_{h f}:=\sigma N \sigma^{-1}$ where $\sigma$ is the permutation corresponding to the rotated English filling of $\lambda$, equipped with the $S^{1}$-action 
defined in (7). Then the inclusion $\iota: \mathcal{S}_{N_{h f}}^{S^{1}} \hookrightarrow \mathcal{S}_{N_{h f}}$ induces an injection in $S^{1}$-equivariant cohomology

$$
\iota^{*}: H_{S^{1}}^{*}\left(\mathcal{S}_{N_{h f}}\right) \hookrightarrow H_{S^{1}}^{*}\left(\mathcal{S}_{N_{h f}}^{S^{1}}\right) \cong \bigoplus_{w \in \mathcal{S}_{N_{h f}}^{1}} H_{S^{1}}^{*}(\mathrm{pt}) \cong \bigoplus_{w \in \mathcal{S}_{N_{h f}}^{1}} \mathbb{C}[t]
$$

The next result, which we use later, is straightforward.

Proposition 5.8. Let $N, \lambda, \mathcal{S}_{N_{h f}}$ be as above. If the columns of the matrix

$$
\left(p_{\text {roll }(w)}(u)\right)_{w, u \in \mathcal{S}_{N_{h f}}^{1}}
$$

(where the variable $w$ is the index of the columns and $u$ the index of the rows) are linearly independent over $H_{S^{1}}^{*}(\mathrm{pt}) \cong \mathbb{C}[t]$, then the set of Springer Schubert classes $\left\{p_{\text {roll }(w)}\right\}_{w \in \mathcal{S}_{N_{h f}}^{S^{1}}}$ is linearly independent.

Next recall that equivariant Schubert classes satisfy a poset-upper-triangularity condition, by which we mean

$$
\sigma_{v}(w)=0 \text { if } w \nsupseteq v
$$

for all $w, v \in S_{n}$. It follows that if the rolldowns $\operatorname{roll}(w)$ of the Hessenberg fixed points satisfy the poset-upper-triangularity condition

$$
\operatorname{roll}(w) \leq u \Leftrightarrow w \leq u
$$

for all $w, u \in \mathcal{S}_{N_{h f}}^{S^{1}}$, then the corresponding Springer Schubert classes are linearly independent [9, Section 2]. Unfortunately, in the $(n-2,2)$ Springer case studied below, the condition (20) is not satisfied, so we cannot immediately conclude linear independence.

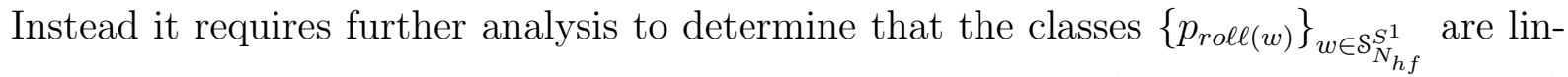
early independent; this is the content of Section 7 below, where (following Proposition 5.8) we check explicitly that the matrix $\left(p_{\text {roll }(w)}(u)\right)_{w, u \in \mathcal{S}_{N_{h f}}^{S 1}}$ has linearly independent columns over $H_{S^{1}}^{*}(\mathrm{pt}) \cong \mathbb{C}[t]$. The poset-upper-triangularity notion is still useful, however, since in our case the failure of (20) is still rather mild, and we see (cf. Question 8.1) that a simple change of coordinates does produce a poset-upper-triangular basis.

Remark 5.9. The $(n-2,2)$ Springer variety example studied here is the first example in the poset pinball literature of an instance of successful Betti pinball which does not yield a poset-upper-triangular basis.

\section{The cases $n=4$ and $n=5$}

In this section and Section 7 we restrict attention to the nilpotent Springer varieties corresponding to Young diagrams of the form $(n-2,2)$ for $n \geq 4$. In this setting we denote by $\mathcal{S}_{(n-2,2)}$ the Springer variety $\mathcal{S}_{N_{h f}}$ corresponding to the nilpotent matrix $N_{h f}:=\sigma N \sigma^{-1}$ 
with associated Young diagram $(n-2,2)$ in the highest form where $\sigma$ is the permutation corresponding to the rotated English filling of $(n-2,2)$. The goal, as explained in Section 5 , is to prove that the dimension pair algorithm produces in this case a module basis for $H_{S^{1}}^{*}\left(\mathcal{S}_{(n-2,2)}\right)$. To this end we concretely compute the Springer fixed points, associated permissible fillings, dimension pairs, and rolldowns for the cases $n=4$ and $n=5$, i.e. for the Springer varieties corresponding to the Young diagrams

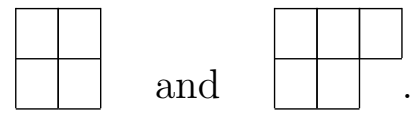

We also explicitly check in these cases that the corresponding Springer Schubert classes are poset-upper-triangular and hence linearly independent. The inductive argument we give in the next section requires the $n=4$ case as its base case. We choose to additionally explicitly compute and record the $n=5$ case because it suggests the outline of the general inductive argument.

Below we present two tables of data. The columns correspond to the following:

- $w$ : an $S^{1}$-fixed point in the Springer variety $\mathcal{S}_{(n-2,2)}$.

- $w^{-1}$ : the inverse of $w$.

- perm filling: the permissible filling $\phi_{\lambda, \sigma}^{-1}\left(w^{-1}\right)$.

- dim pair: the dimension pairs of the permissible filling.

- deg: the number of dimension pairs of the permissible filling (equivalently, the cohomology degree of the associated Springer Schubert class).

- $\omega(\mathbf{x})$ : the permutation associated to the list $\mathbf{x}$ of top parts of the dimension pairs.

- $\operatorname{rol\ell }(w)$ : inverse of $\omega(\mathbf{x})$, and by definition of the dimension pair algorithm, the rolldown of $w$.

Example 6.1. Let $n=4$ and $\lambda_{N}=(2,2)$. The following table records the data outlined above. Part of these computations are also contained in [13].

From this table it can be seen explicitly that the only Springer fixed point $w$ in $\mathcal{S}_{(2,2)}$ with $\operatorname{roll}(w) \neq w$ is $w=2413$. Moreover it is straightforward to check that the rolldown 1423 of $w=2413$ is not Bruhat-less than any of the other Springer fixed points. These facts together imply that these Springer fixed points and associated rolldowns satisfy the poset-upper-triangularity property

$$
\operatorname{roll}(w) \leq u \Leftrightarrow w \leq u
$$

for all fixed points $w, u$. By an argument identical to [2, Lemma 4.4] which uses the poset-upper-triangularity property (19) of the equivariant Schubert classes $\left\{\sigma_{w}\right\}_{w \in S_{n}}$, this

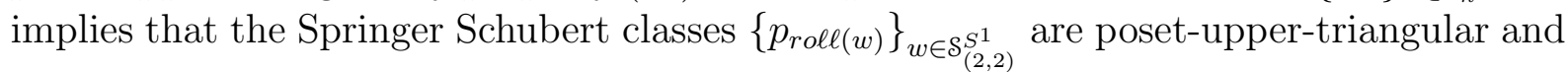
hence linearly independent and a $H_{S^{1}}^{*}(\mathrm{pt})$-module basis for $H_{S^{1}}^{*}\left(\mathcal{S}_{(2,2)}\right)$. 
Table 1: Dimension pair data for the Springer variety $\mathcal{S}_{(2,2)}$.

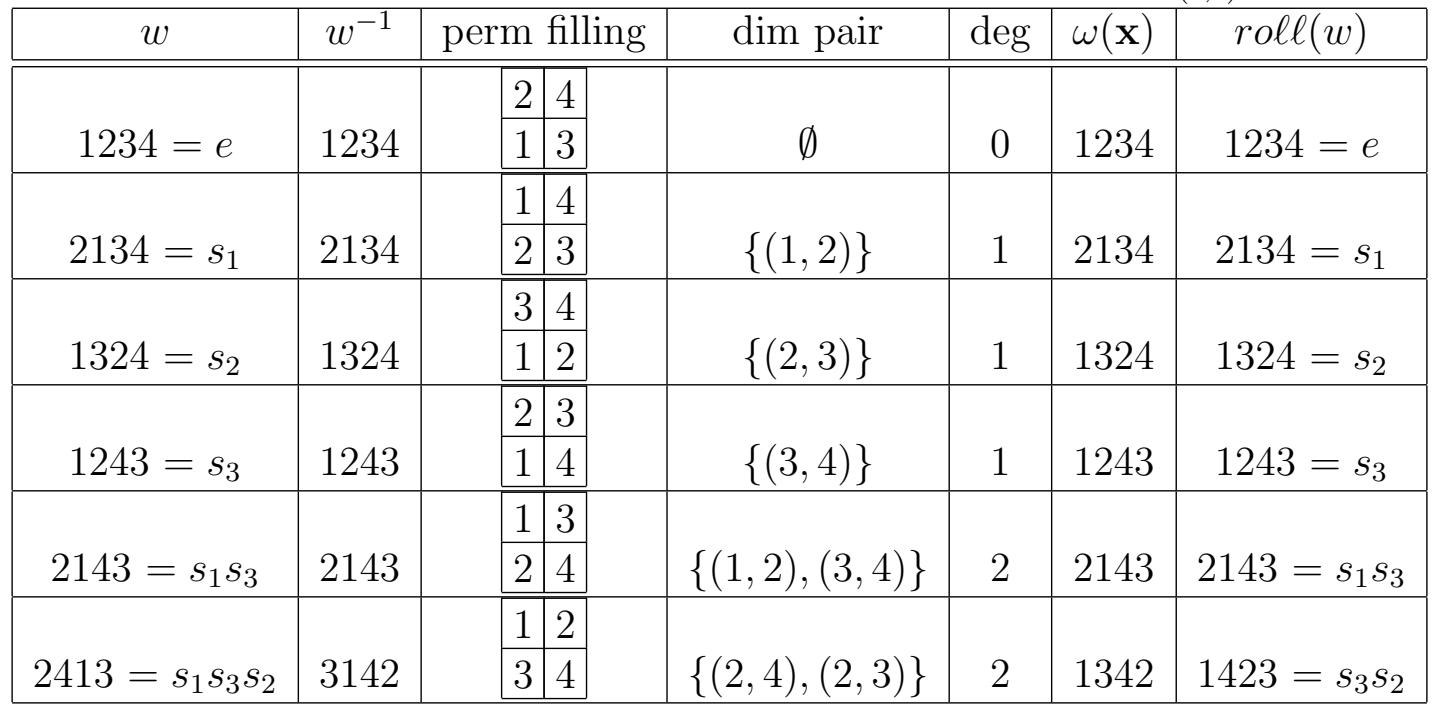

We have just explicitly checked that in the case $n=4$, the dimension pair algorithm interpreted in terms of Betti pinball produces a module basis of $H_{S^{1}}^{*}\left(\mathcal{S}_{(2,2)}\right)$. We now compute the $n=5$ case and relate it to the $n=4$ case, thereby illustrating the outline of the general inductive argument.

Example 6.2. Let $n=5$ and $\lambda=(3,2)$. Suppose $T$ is a permissible filling of $(3,2)$ where the entry 5 is in the top row. Since the rows in a permissible filling are increasing this means that the 5 occurs in the rightmost box of the top row of $T$. Deleting this box yields a valid permissible filling of $(2,2)$ which therefore occurs in the previous $n=4$ example. For permissible fillings $T$ of this form the corresponding fixed point $w$ and its rolldown are easily seen to be identical to those obtained in the previous example (viewed as elements of $S_{5}$ instead of $S_{4}$ via the usual embedding $S_{4} \hookrightarrow S_{5}$ ). Hence the permissible fillings in the $n=5$ case which do not occur in the $n=4$ case are precisely those for which the entry 5 is in the bottom row. There are four such permissible fillings as may be seen in the table below.

We claim that, as in the $n=4$ case, the rolldowns satisfy the condition (21), which then implies by the same argument that the corresponding Springer Schubert classes are poset-upper-triangular and hence linearly independent and a module basis. To prove this claim it suffices to check $(21)$ for those $w$ for which $\operatorname{rol\ell }(w) \neq w$. We check each case by hand.

For $w=s_{1} s_{3} s_{2}$ with $\operatorname{roll}(w)=s_{3} s_{2}$, we see that $\operatorname{roll}(w)<s_{3} s_{4} s_{1} s_{2}$ and also that $\operatorname{roll}(w)<s_{3} s_{4} s_{1} s_{2} s_{3}$. Since also $w<s_{3} s_{4} s_{1} s_{2}$ and $w<s_{3} s_{4} s_{1} s_{2} s_{3}$, the claim holds in this case. Next observe that the last four fixed points in the above table are linearly ordered with respect to the Bruhat order, i.e.

$$
s_{3} s_{4}<s_{3} s_{4} s_{1}<s_{3} s_{4} s_{1} s_{2}<s_{3} s_{4} s_{1} s_{2} s_{3}
$$

In the case of $w=s_{3} s_{4}$ we have $\operatorname{roll}(w)=s_{4}$. Moreover $s_{4}$ is not Bruhat-less than any 
Table 2: Dimension pair data for the Springer variety $\mathcal{S}_{(3,2)}$.

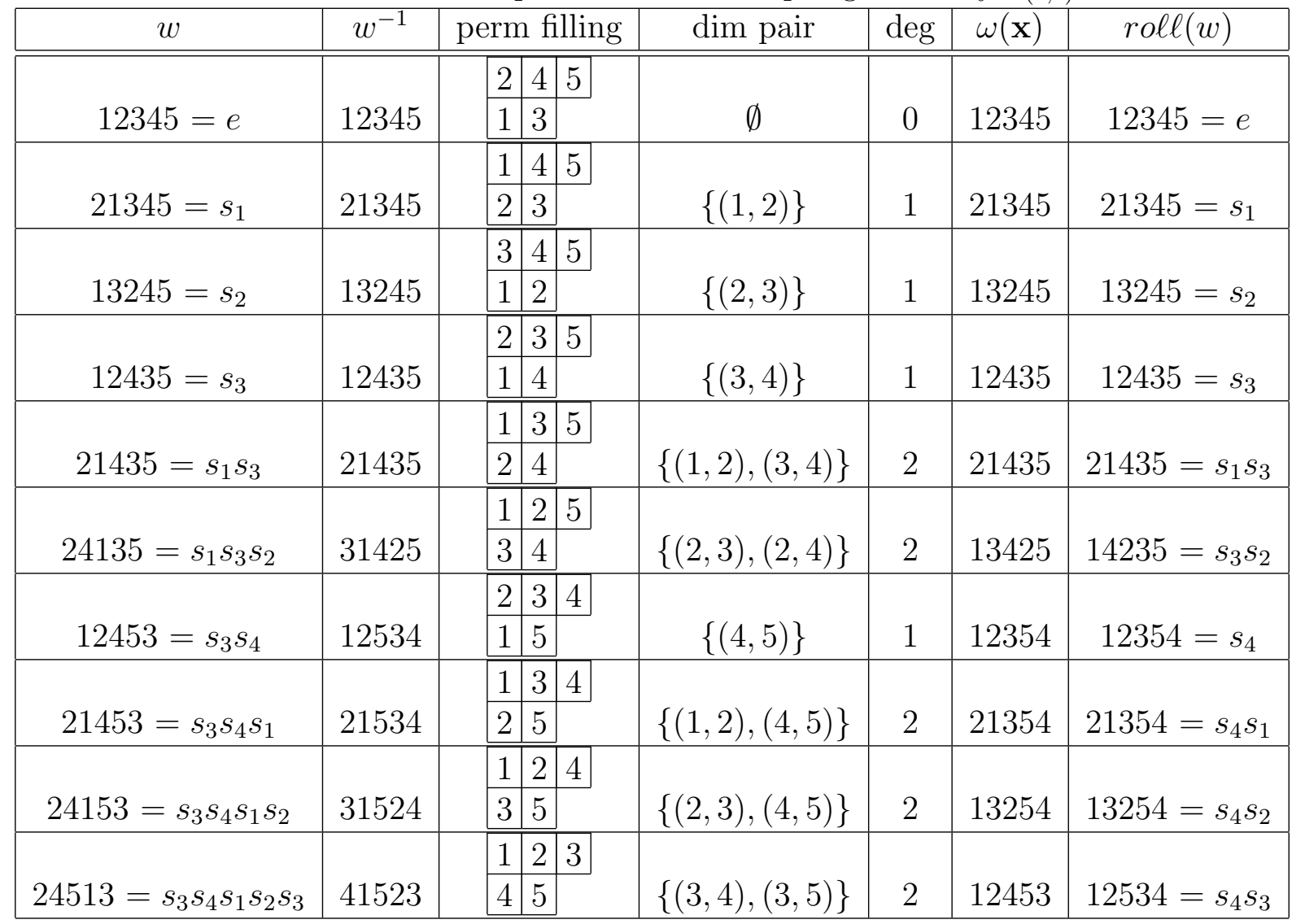

of the fixed points occurring in the $n=4$ case and is Bruhat-less than all of the last four fixed points, so the claim holds in this case. Similarly, the rolldowns for the last three fixed points satisfy

$$
\begin{aligned}
& \operatorname{roll}\left(s_{3} s_{4} s_{1}\right)=s_{4} s_{1} \nless s_{3} s_{4}, \\
& \operatorname{roll}\left(s_{3} s_{4} s_{1} s_{2}\right)=s_{4} s_{2} \nless s_{3} s_{4} s_{1}, \\
& \operatorname{roll}\left(s_{3} s_{4} s_{1} s_{2} s_{3}\right)=s_{4} s_{3} \nless s_{3} s_{4} s_{1} s_{2},
\end{aligned}
$$

so the claim holds in all cases. This proves the claim and hence that the Springer Schubert classes are poset-upper-triangular in the $n=5$ case and hence a module basis, as desired.

\section{A poset pinball module basis for $(n-2,2)$ Springer varieties}

The main result of this section is that the dimension pair algorithm produces a set of

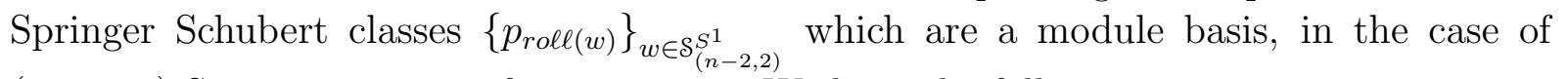
$(n-2,2)$ Springer varieties for any $n \geq 4$. We have the following. 
Theorem 7.1. Let $n \geq 4$. Let $N: \mathbb{C}^{n} \rightarrow \mathbb{C}^{n}$ be a nilpotent operator in Jordan canonical form with weakly decreasing Jordan block sizes $n-2$ and 2 . Let $N_{h f}:=\sigma N \sigma^{-1}$ be the choice of the highest form of $N$ where $\sigma$ is the permutation corresponding to the rotated English filling of $(n-2,2)$. Let $\mathcal{S}_{(n-2,2)}$ be the Springer variety corresponding to $N_{h f}$ equipped with the $S^{1}$-action defined in (7). Let roll $: \mathcal{S}_{(n-2,2)}^{S^{1}} \rightarrow S_{n}$ be the function defined by the dimension-pair algorithm. Then the columns of the matrix

$$
\left(p_{\text {roll }(w)}(u)\right)_{w, u \in \mathcal{S}_{(n-2,2)}^{S^{1}}}
$$

with entries in $H_{S^{1}}^{*}(\mathrm{pt}) \cong \mathbb{C}[t]$ are linearly independent over $H_{S^{1}}^{*}(\mathrm{pt})$. (Here $w$ is the variable indexing the columns and $u$ the index of the rows.) In particular, the Springer

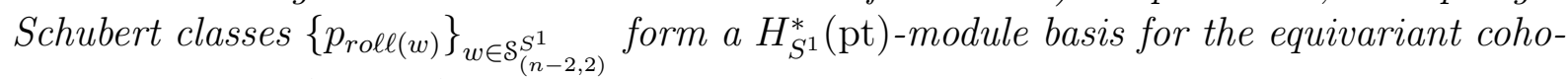
mology ring $H_{S^{1}}^{*}\left(\mathcal{S}_{(n-2,2)}\right)$ of the Springer variety.

Remark 7.2. The above theorem extends the subregular Springer case (which corresponds to Young diagrams of shape $(n-1,1))$, for which it was shown in [9] that the set of Springer Schubert classes obtained by the dimension pair algorithm is poset-upper-triangular, so in particular linearly independent. (Although the results in [9] are not phrased using the terminology of this paper it is straightforward to see that the classes used in [9] agree with those arising from the dimension pair algorithm.)

Since the rows are increasing in a Springer permissible filling, we can naturally decompose the set of $(n-2,2)$ permissible fillings into two subsets: namely, those for which the largest entry $n$ occupies the top row, and those for which $n$ occupies the bottom row. As observed in Example 6.2 above, when $n$ is in the top row, the permissible filling obtained by removing the rightmost box in the top row is a permissible filling for the Young diagram $(n-3,2)$, corresponding to the smaller Springer variety $\mathcal{S}_{(n-3,2)}$. This sets us up for an inductive argument. Since we have already seen in Section 6 the linear independence for the cases $n=4$ and $n=5$, we start the induction at $n=6$. We begin with a preliminary lemma generalizing the observations made in Example 6.2, for which we refer to the table given below.

Lemma 7.3. Let $n \geq 6$. Let $N, N_{h f}, \mathcal{S}_{(n-2,2)}$ and roll be as in Theorem 7.1. Then

- there are precisely $n-1$ permissible fillings of $(n-2,2)$ with $n$ in the bottom row,

- the $n-1$ such permissible fillings, their corresponding Springer fixed points $w$, and their rolldowns roll $(w)$ are precisely those listed in the table,

- these n-1 Springer fixed points are linearly ordered with respect to Bruhat order, i.e.

$s_{3} s_{4} \cdots s_{n-2} s_{n-1}<s_{3} s_{4} \cdots s_{n-2} s_{n-1} s_{1}<\cdots<s_{3} s_{4} \cdots s_{n-2} s_{n-1} s_{1} s_{2} \cdots s_{n-3} s_{n-2}$.

Proof of Lemma 7.3. Since the Springer permissible fillings are precisely those which are row-strict, it is immediate that the permissible fillings listed in the table are precisely 
Table 3: Dimension pair algorithm data for the Springer fixed points in $\mathcal{S}_{(n-2,2)}^{S^{1}}$ corresponding to permissible fillings with $n$ in the bottom row.

\begin{tabular}{|c|c|c|c|c|c|c|}
\hline \multicolumn{4}{|r|}{$\mathrm{pf}$} & $w^{-1}$ & $w$ & $v_{h f}$ \\
\hline 2 & 23 & 4 & \begin{tabular}{c|c|}
$\cdots$ & $n-1$ \\
\end{tabular} & \multirow[b]{2}{*}{$12 n 34 \cdots$} & \multirow[b]{2}{*}{$s_{3} s_{4} \cdots s_{n-2} s_{n-1}$} & \multirow[b]{2}{*}{$s_{n-1}$} \\
\hline 1 & $\begin{array}{ll}1 & n \\
\end{array}$ & & & & & \\
\hline 1 & $\begin{array}{ll}1 & 3 \\
\end{array}$ & 4 & \begin{tabular}{l|l|}
$\cdots$ & $n-1$ \\
\end{tabular} & \multirow[b]{2}{*}{$21 n 34 \cdots$} & \multirow[b]{2}{*}{$s_{3} s_{4} \cdots s_{n-2} s_{n-1} s_{1}$} & \multirow[b]{2}{*}{$s_{n-1} s_{1}$} \\
\hline 2 & $2 n$ & & & & & \\
\hline 1 & $1 \quad 2$ & 4 & $n-1$ & \multirow[b]{2}{*}{$31 n 24 \cdots$} & \multirow[b]{2}{*}{$s_{3} s_{4} \cdots s_{n-2} s_{n-1} s_{1} s_{2}$} & \multirow[b]{2}{*}{$s_{n-1} s_{2}$} \\
\hline 3 & $\begin{array}{ll}3 & n \\
\end{array}$ & & & & & \\
\hline 1 & \begin{tabular}{l|l}
1 & 2 \\
\end{tabular} & 3 & \begin{tabular}{l|l|}
$\cdots$ & $n-1$ \\
\end{tabular} & \multirow[b]{2}{*}{$41 n 23 \cdots$} & \multirow[b]{2}{*}{$s_{3} s_{4} \cdots s_{n-2} s_{n-1} s_{1} s_{2} s_{3}$} & \multirow[b]{2}{*}{$s_{n-1} s_{3}$} \\
\hline & \begin{tabular}{l|l}
4 & $n$ \\
\end{tabular} & & & & & \\
\hline \multicolumn{4}{|r|}{$\vdots$} & $\vdots$ & $\vdots$ & $\vdots$ \\
\hline 1 & 2 & $\ldots$ & \begin{tabular}{l|l|l|}
$\cdot \cdot$ & $n-3$ & $n-2$ \\
\end{tabular} & \multirow[b]{2}{*}{$n-11 n 23 \cdots$} & \multirow[b]{2}{*}{$\begin{array}{c}s_{3} s_{4} \cdots s_{n-2} s_{n-1} \\
s_{1} s_{2} \cdots s_{n-3} s_{n-2}\end{array}$} & \multirow[b]{2}{*}{$s_{n-1} s_{n-2}$} \\
\hline$n-1$ & \begin{tabular}{l|l}
1 & $n$ \\
\end{tabular} & & & & & \\
\hline
\end{tabular}

those with $n$ in the bottom row. In particular there are exactly $n-1$ such permissible fillings as claimed. Moreover, it follows from the definition of $\phi_{\lambda, \sigma}$ (which corresponds to the rotated English reading) that the one-line notation of the $w^{-1}$ are those given in the table. Explicit computation also verifies that the following expressions in the simple transpositions are indeed reduced word decompositions of the $w^{-1}$ :

- $12 n 34 \cdots=s_{n-1} s_{n-2} \cdots s_{4} s_{3}$

- $21 n 34 \cdots=s_{1} s_{n-1} s_{n-2} \cdots s_{4} s_{3}$

- $31 n 24 \cdots=s_{2} s_{1} s_{n-1} s_{n-2} \cdots s_{4} s_{3}$

- $41 n 23 \cdots=s_{3} s_{2} s_{1} s_{n-1} s_{n-2} \cdots s_{4} s_{3}$

and so on, up to

- $n-11 n 23 \cdots=s_{n-2} s_{n-1} \cdots s_{2} s_{1} s_{n-1} s_{n-2} \cdots s_{4} s_{3}$,

from which it follows that the $w$ are those given in the list. For $k$ with $1 \leq k \leq n-1$, the definition of dimension pairs implies that the permissible filling with $k$ and $n$ in the bottom row contains as dimension pairs $\{(k-1, k),(n-1, n)\}$ for $2 \leq k \leq n-1$ and $\{(n-1, n)\}$ for $k=1$. From this it follows from the definition of $\omega(\mathbf{x})$ that $\operatorname{rol} \ell(w)$ is as given in the table. Finally, from the given reduced word decompositions and the definition of Bruhat order we obtain (22) as desired.

Before proceeding with the proof of Theorem 7.1 we briefly recall the Billey formula for computing restrictions of Schubert classes. For the purposes of this discussion only, let $w, u$ denote arbitrary elements in $S_{n}$. Billey's formula gives a computation of the restriction $\sigma_{w}(u)$ of the class $\sigma_{w}$ at $u$. We use the formulation given in [11]. Let $\alpha_{i}$ denote the simple root $t_{i}-t_{i+1}$ and $\widehat{\alpha}_{i}$ the operator on $H_{\mathbb{T}}^{*}(\mathrm{pt})$ which multiplies by $\alpha_{i}$. 
Theorem 7.4. ([3, Theorem 4], also cf. [11]) Suppose I is a reduced word expression for $u \in S_{n}$. For each $w \in S_{n}$ we have

$$
\sigma_{w}(u)=\sum_{J \subseteq I} \prod_{i \in I}\left(\widehat{\alpha}_{i}^{[i \in J]} r_{i}\right) \cdot 1
$$

where the sum is over reduced subwords $J$ of I with product $w$, the notation $\widehat{\alpha}_{i}^{[i \in J]}$ means that $\widehat{\alpha}_{i}$ is included only if $i \in J$, and $r_{i}$ is the reflection corresponding to $s_{i}$.

We record the following fact, used in the proof below, which follows straightforwardly from the Billey formula.

Fact 7.5. Suppose $w, u \in S_{n}$ with $w \leq u$ in Bruhat order. Suppose there exists a decomposition $u=u^{\prime} \cdot u^{\prime \prime}$ for $u^{\prime}, u^{\prime \prime} \in S_{n}$ where $w \leq u^{\prime}$ and, for all simple transpositions $s_{i}$ such that $s_{i}<w$, we have $s_{i} \not \leq u^{\prime \prime}$. Then $\sigma_{w}(u)=\sigma_{w}\left(u^{\prime}\right)$.

Having recalled the Billey formula, for the remainder of this discussion we will use the letters $w, u$ to denote Springer fixed points. Recall (cf. Section 5) that we wish to

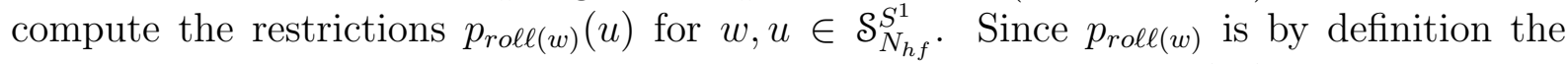
image of the equivariant Schubert class $\sigma_{\text {roll }(w)}$ under the ring map (18) and because the diagram

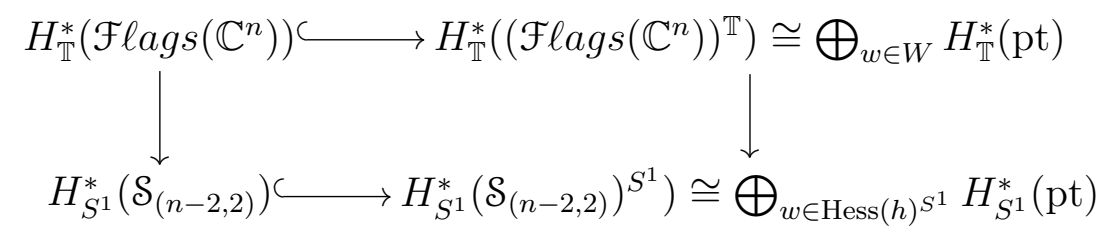

commutes, the polynomial $p_{\text {roll }(w)}(u) \in H_{S^{1}}^{*}(\mathrm{pt}) \cong \mathbb{C}[t]$ can be computed by first evaluat-

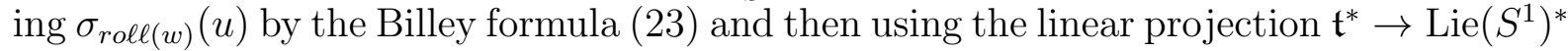
for our choice of $S^{1}$ in (7) given in Lemma 3.29. We use this technique repeatedly in the proof below.

Proof of Theorem 7.1. By Propositions 5.7 and 5.8, it suffices to prove that the matrix obtained from the restrictions to fixed points

$$
\left(p_{r o \ell \ell(w)}(u)\right)_{w, u \in S_{(n-2,2)}^{11}}
$$

has $H_{S^{1}}^{*}(\mathrm{pt})$-linearly independent columns.

Let $n \geq 4$. We have seen in Section 6 that the above assertion holds for the cases $n=4$ and $n=5$. Hence assume now that $n \geq 6$. We assume by induction that for the $n-1$ case, i.e. for the case of the partition $(n-3,2)$, the above matrix has linearly independent columns.

For concreteness and for the remainder of the argument, we assume that the fixed points $w \in \mathcal{S}_{(n-2,2)}^{S^{1}}$ have been linearly ordered so that the fixed points corresponding to permissible fillings containing the $n$ in the top row appear first, and that the fixed points associated to fillings with $n$ in the bottom row are given the ordering in the table in 
Lemma 7.3 (reading from top to bottom). Ordered in this manner, we may write the above matrix in terms of submatrices as follows:

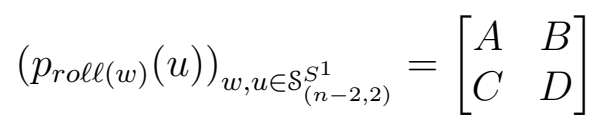

where the submatrix $A$ has entries $p_{\text {roll }(w)}(u)$ where both $w, u$ correspond to fillings with $n$ in the top row, $D$ corresponds to those where both $w, u$ have $n$ in the bottom row, and so on.

Consider the submatrix $A$. For an entry $p_{\text {roll }(w)}(u)$ in $A$, by assumption $w$ is in the subgroup $S_{n-1} \subseteq S_{n}$ and it is straightforward to see from the definition of the dimension pair algorithm that $\operatorname{rol\ell }(w)$ is equal to the rolldown of $w$ considered as an element of $\mathcal{S}_{(n-3,2)}^{S^{1}}$. Since $u \in S_{n-1}$ also this submatrix is equal to the matrix of restrictions to fixed points obtained in the $(n-3,2)$ case and so by induction $A$ has linearly independent columns.

Next consider the submatrix $B$ corresponding to $p_{\text {roll }(w)}(u)$ where $\phi_{\lambda, \sigma}^{-1}\left(w^{-1}\right)$ has $n$ in the bottom row and $\phi_{\lambda, \sigma}^{-1}\left(u^{-1}\right)$ has $n$ in the top row. From Lemma 7.3 and the table given there, we know that the rolldown $\operatorname{roll}(w)$ of any such $w$ contains the simple transposition $s_{n-1}$ in its reduced word decomposition. On the other hand, for $u$ with $n$ in the top row, $u$ is an element in the subgroup $S_{n-1}$ which fixes the element $n$, and in particular a reduced word decomposition for $u$ may be written solely with the simple transpositions $s_{1}, s_{2}, \ldots, s_{n-2}$. Hence $\operatorname{rol\ell }(w) \not \leq u$ in Bruhat order, and by the upper-triangularity

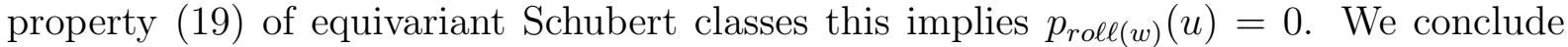
that the entire submatrix is 0 and the matrix (24) is in fact of the form

$$
\left[\begin{array}{ll}
A & 0 \\
C & D
\end{array}\right]
$$

where $A$ has linearly independent columns. In order to prove that the full matrix has linearly independent columns, we wish to prove that the submatrix $D$ has linearly independent columns. The remainder of the proof is dedicated to the justification of this last

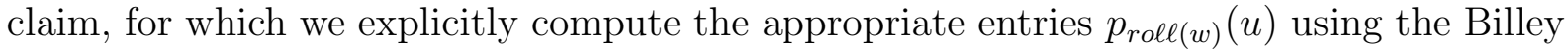
formula (23).

We compute each column of $D$ in the linear order given by the enumeration in Lemma 7.3 of those $w$ with $n$ in the bottom row. For the Billey computations below we use the choices of reduced word decompositions for $w$ and $\operatorname{rol\ell }(w)$ given in the same lemma.

First consider the case $w=s_{3} s_{4} \cdots s_{n-2} s_{n-1}$. Then $\operatorname{roll}(w)=s_{n-1}$. We claim $\sigma_{s_{n-1}}$ evaluates to $t_{3}-t_{n}$ at all fixed points $u$. Indeed, recalling that the reflection $r_{i}$ acts on the variables $t_{j}$ by $r_{i}\left(t_{i}\right)=t_{i+1}, r_{i}\left(t_{i+1}\right)=t_{i}$ and $r_{i}\left(t_{j}\right)=t_{j}$ for all $j \neq i, i+1$, we have for instance

$$
\begin{aligned}
\sigma_{s_{n-1}}\left(s_{3} s_{4} \cdots s_{n-2} s_{n-1}\right) & =r_{3} r_{4} \cdots r_{n-2}\left(t_{n-1}-t_{n}\right) \\
& =r_{3} r_{4} \cdots r_{n-3}\left(t_{n-2}-t_{n}\right) \\
& \vdots \\
& =t_{3}-t_{n},
\end{aligned}
$$


which proves the claim for $u=w$. For all other $u$ with $n$ in the bottom row, the computation of the Billey formula differs from (25) only in that there are extra simple transpositions occurring after the $s_{n-1}$ in the reduced word decomposition of $u$. By Fact 7.5 these extra transpositions make no difference in the evaluation of $\sigma_{s_{n-1}}(u)$ and

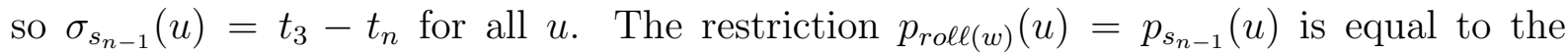
image of $\sigma_{s_{n-1}}(u) \in H_{\mathbb{T}}^{*}(\mathrm{pt})$ under the projection map $H_{\mathbb{T}}^{*}(\mathrm{pt}) \rightarrow H_{S^{1}}^{*}(\mathrm{pt})$ induced from the inclusion $S^{1} \hookrightarrow \mathbb{T}$. By Lemma 3.29 we know $t_{3} \mapsto t$ and $t_{n} \mapsto(n+3-n) t=3 t$ under this projection, from which we conclude that the first (leftmost) column of $D$ is

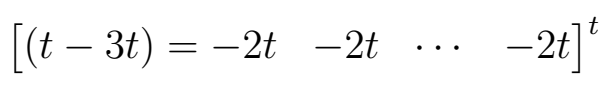

where we have written in transposed form for typographical convenience.

For the next several cases, the computations are similar to the example above, so we keep explanation brief. Consider the case $w=s_{3} s_{4} \cdots s_{n-2} s_{n-1} s_{1}$ and $\operatorname{rol\ell }(w)=s_{n-1} s_{1}$. In this case, $\sigma_{s_{n-1} s_{1}}\left(s_{3} s_{4} \cdots s_{n-2} s_{n-1}\right)=0$ since $s_{n-1} s_{1}$ does not occur as a subword of $s_{3} s_{4} \cdots s_{n-2} s_{n-1}$. Also, $\sigma_{s_{n-1} s_{1}}$ evaluates to $\left(t_{3}-t_{n}\right)\left(t_{1}-t_{2}\right)$ at all other $u$. Thus the column corresponding to this $w$ is

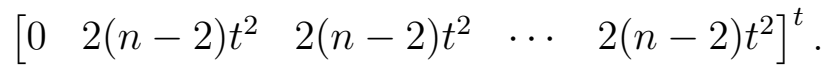

Next consider the case $w=s_{3} s_{4} \cdots s_{n-2} s_{n-1} s_{1} s_{2}$ and $\operatorname{rol\ell }(w)=s_{n-1} s_{2}$. In this case

$$
\sigma_{s_{n-1} s_{2}}\left(s_{3} s_{4} \cdots s_{n-2} s_{n-1}\right)=\sigma_{s_{n-1} s_{2}}\left(s_{3} s_{4} \cdots s_{n-2} s_{n-1} s_{1}\right)=0
$$

since there are no reduced subwords in $s_{3} s_{4} \cdots s_{n-2} s_{n-1}$ equal to $\operatorname{roll}(w)=s_{n-1} s_{2}$. Furthermore, $\sigma_{s_{n-1} s_{2}}$ evaluates to $\left(t_{1}-t_{4}\right)\left(t_{3}-t_{n}\right)$ on all other $u$. Hence the column corresponding to this $w$ is

$$
\left[\begin{array}{lllll}
0 & 0 & 2(n-3) t^{2} & \cdots & 2(n-3) t^{2}
\end{array}\right]^{t} .
$$

Next consider the case $w=s_{3} s_{4} \cdots s_{n-2} s_{n-1} s_{1} s_{2} s_{3}$ where $\operatorname{roll}(w)$ is $s_{n-1} s_{3}$. In this case, $\sigma_{s_{n-1} s_{3}}$ evaluates to $\left(t_{3}-t_{4}\right)\left(t_{3}-t_{n}\right)$ on the first 3 fixed points listed in the table in Lemma 7.3. Moreover $\sigma_{s_{n-1} s_{3}}$ evaluates to $\left(t_{3}-t_{4}\right)\left(t_{3}-t_{n}\right)+\left(t_{1}-t_{5}\right)\left(t_{3}-t_{n}\right)$ at all other $u$. Thus the column corresponding to this $w$ is

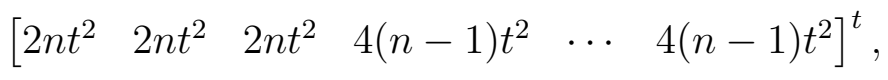

where there are $(n-1)-3=n-4$ entries of the form $4(n-1) t^{2}$.

For the next case, suppose $n \geq 7$. (In the special case $n=6$, this case is vacuous.) Suppose $k \in \mathbb{Z}$ with $4 \leq k \leq n-3$. Let $w=s_{3} s_{4} \cdots s_{n-2} s_{n-1} s_{1} s_{2} \cdots s_{k-1} s_{k}$ and $\operatorname{roll}(w)=s_{n+1} s_{k}$. By assumption on $k$, the simple transposition $s_{k}$ commutes with $s_{n-1}$. In this case $\sigma_{s_{n-1} s_{k}}$ evaluates to $\left(t_{3}-t_{k+1}\right)\left(t_{3}-t_{n}\right)$ on all fixed points listed in Lemma 7.3 up to $s_{3} s_{4} \cdots s_{n-2} s_{n-1} s_{1} s_{2} \cdots s_{k-1}$. There are $k$ fixed points in all of this form. Moreover, $\sigma_{s_{n-1} s_{k}}$ evaluates to $\left(t_{3}-t_{k+1}\right)\left(t_{3}-t_{n}\right)+\left(t_{1}-t_{k+2}\right)\left(t_{3}-t_{n}\right)$ on the remaining fixed points $u$ which contain $s_{3} s_{4} \cdots s_{n-2} s_{n-1} s_{1} s_{2} \cdots s_{k-1} s_{k}$. Hence when projected to $H_{S^{1}}^{*}(\mathrm{pt})$, the 
column corresponding to such a $w$ is

$$
\left[\begin{array}{c}
2(n-k+3) t^{2} \\
\vdots \\
2(n-k+3) t^{2} \\
(2(n-k+3)+2(n-k+1)) t^{2} \\
\vdots \\
(2(n-k+3)+2(n-k+1)) t^{2}
\end{array}\right]
$$

where there are $k$ entries of the form $2(n-k+3) t^{2}$ and $n-1-k$ entries of the form $(2(n-k+3)+2(n-k+1)) t^{2}$.

Finally, consider the case $w=s_{3} s_{4} \cdots s_{n-2} s_{n-1} s_{1} s_{2} \ldots s_{n-3} s_{n-2}$ and $\operatorname{roll}(w)=s_{n-1} s_{n-2}$. Since $s_{n-1}$ and $s_{n-2}$ do not commute, this computation is somewhat different from the ones given above; in particular roll $(w)$ is not Bruhat-less than any of the fixed points $u$ except for the last one listed in Lemma 7.3. Hence in this case $\sigma_{s_{n-1} s_{n-2}}(u)=0$ at all $u$ except for $u=s_{3} s_{4} \cdots s_{n-2} s_{n-1} s_{1} s_{2} \cdots s_{n-3} s_{n-2}$, and at this last $u$, we can compute

$$
\sigma_{s_{n-1} s_{n}}\left(s_{3} s_{4} \cdots s_{n-2} s_{n-1} s_{1} s_{2} \cdots s_{n-3} s_{n-2}\right)=\left(t_{3}-t_{n}\right)\left(t_{1}-t_{n}\right) \text {. }
$$

Hence the column corresponding to this last $w$ is

$$
\left[\begin{array}{lllll}
0 & 0 & \cdots & 0 & 2 t^{2}
\end{array}\right]^{t} .
$$

We now prove that the columns $p_{w}$ for $w$ as above are linearly independent over the ring $H_{S^{1}}^{*}(\mathrm{pt}) \cong \mathbb{C}[t]$. The first column $p_{w}$ with $w=s_{3} s_{4} \cdots s_{n-2} s_{n-1}$ has a $-2 t$ in each entry. We may add or subtract any multiple of this column to or from any other column, and if the resulting set of columns is linearly independent, then so is the original set of columns. It is straightforward to check that for all $k$ with $3 \leq k \leq n-1$, subtracting $2(n-k+3)$ times the first column from the column corresponding to $w$ with rolldown $\operatorname{rol\ell }(w)=s_{n-1} s_{k}$ yields

$$
\left[\begin{array}{llllll}
0 & \cdots & 0 & 2(n-k+1) t^{2} & \cdots & 2(n-k+1) t^{2}
\end{array}\right]^{t}
$$

where there are $k$ zeroes at the top of the column and $(n-1)-k$ entries at the bottom of the form $2(n-k+1) t^{2}$. In particular, adjusted in this manner, the resulting matrix is lower-triangular with non-zero entries along the diagonal, so its columns are linearly independent. As argued above, this implies that the matrix $D$ has linearly independent columns, as was desired. This completes the proof.

\section{Open questions}

We close with some open questions for future work. 
Question 8.1. The computations in the proof of Theorem 7.1 explicitly show that the

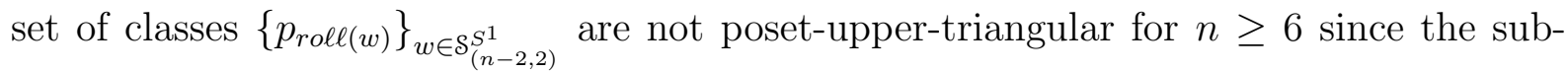
matrix $D$ discussed in the proof has non-zero entries both above and below its main diagonal. However the proof also shows that a simple change of basis does yield a posetupper-triangular basis, the elements of which are linear combinations of Springer Schubert classes. We do not know whether this is an instance of a more general phenomenon. It would be of interest to clarify the situation for other cases of Springer varieties.

Question 8.2. Both Tymoczko's paving by affines of Hessenberg varieties and the interpretation of our dimension pair algorithm via poset pinball depend on using a Hessenberg variety $\operatorname{Hess}(N, h)$ for which the nilpotent operator $N$ is in a highest form. In the case of Tymoczko's paving, this choice can be viewed as a matter only of convenience in the sense that any other translated Hessenberg variety $\operatorname{Hess}\left(\sigma N \sigma^{-1}, h\right)$ can be given a paving simply by using translated Schubert cells $\sigma \cdot B w B$ instead of the usual Schubert cells $B w B$. On the other hand, the poset pinball game delicately depends on the choice of initial subset

$$
\operatorname{Hess}(N, h)^{S^{1}} \subseteq S_{n}
$$

Although the sets $\operatorname{Hess}(N, h)^{S^{1}}$ and $\operatorname{Hess}\left(\sigma N \sigma^{-1}, h\right)^{S^{1}}$ are also related by a simple translation by $\sigma$, multiplication by a permutation does not preserve Bruhat order, so pinball results do not immediately translate from $\operatorname{Hess}(N, h)$ to $\operatorname{Hess}\left(\sigma N \sigma^{-1}, h\right)$. One of the main results of this manuscript is that, for a certain special family of Hessenberg varieties $\operatorname{Hess}(N, h)=\mathcal{S}_{N_{h f}}$ (where $N_{h f}$ is a particular choice of a highest form) we can use the poset pinball and the dimension pair algorithm to obtain a module basis for $H_{S^{1}}^{*}\left(\mathcal{S}_{N_{h f}}\right)$.

1. It seems plausible that there may be other choices of highest forms (cf. Theorem 3.21), different from that used in this manuscript, which are particularly wellsuited for poset pinball.

2. Furthermore, among the choices of highest forms which behave well for poset pinball, there may also be choices best suited for further applications of pinball bases. More specifically, there may be choices of highest forms $N_{T}$ such that a pinball basis for $H_{S^{1}}^{*}\left(\mathcal{S}_{N_{T}}\right)$ has good properties when mapped to $H_{S^{1}}^{*}\left(\mathcal{S}_{N_{T}}^{S^{1}}\right)$. Such choices could then prove useful for e.g. constructions of representations on equivariant cohomology (analogous to the lifts of the classical Springer representations constructed via pinball in [9]).

\section{References}

[1] D. Bayegan and M. Harada. A Giambelli formula for the $S^{1}$-equivariant cohomology of type $A$ Peterson varieties, arXiv:1012.4053.

[2] D. Bayegan and M. Harada. Poset pinball and type $A$ regular nilpotent Hessenberg varieties, arXiv:1012.4054. 
[3] S. Billey. Kostant polynomials and the cohomology ring of G/B. Duke Math. J., 96:205-224, 1999.

[4] M. Brion and J. B. Carrell. The equivariant cohomology ring of regular varieties. Michigan Math. J., 52(1):189-203, 2004.

[5] J. B. Carrell and K. Kaveh. On the equivariant cohomology of subvarieties of a B-regular variety. Transform. Groups, 13(3-4):495-505, 2008.

[6] F. De Mari, C. Procesi, and M. A. Shayman. Hessenberg varieties. Trans. Amer. Math. Soc., 332(2):529-534, 1992.

[7] J. Fulman. Descent identities, Hessenberg varieties, and the Weil conjectures. J. Combin. Theory Ser. A, 87(2):390-397, 1999.

[8] F. Y. C. Fung. On the topology of components of some Springer fibers and their relation to Kazhdan-Lusztig theory. Adv. Math., 178(2):244-276, 2003.

[9] M. Harada and J. Tymoczko. Poset pinball, GKM-compatible subspaces, and Hessenberg varieties, arXiv:1007.2750.

[10] M. Harada and J. Tymoczko. A positive Monk formula in the $S^{1}$-equivariant cohomology of type A Peterson varieties, arXiv:0908.3517. To be published in Proc.London Math. Soc.

[11] A. Knutson. A Schubert calculus recurrence from the noncomplex $W$-action on $G / B$, June 2003, arXiv: 0306304.

[12] B. Kostant. Flag manifold quantum cohomology, the Toda lattice, and the representation with highest weight $\rho$. Selecta Math. (N.S.), 2(1):43-91, 1996.

[13] A. Mbirika. A Hessenberg generalization of the Garsia-Procesi basis for the cohomology ring of Springer varieties. Electronic Journal of Combinatorics, 17 (electronic), 2010.

[14] K. Rietsch. Totally positive Toeplitz matrices and quantum cohomology of partial flag varieties. J. Amer. Math. Soc., 16(2):363-392 (electronic), 2003.

[15] N. Shimomura. The fixed point subvarieties of unipotent transformations on the flag varieties. J. Math. Soc. Japan, 37(3):537-556, 1985.

[16] N. Spaltenstein. The fixed point set of a unipotent transformation on the flag manifold. Nederl. Akad. Wetensch. Proc. Ser. A 79=Indag. Math., 38(5):452-456, 1976.

[17] J. S. Tymoczko. Linear conditions imposed on flag varieties. Amer. J. Math., 128(6):1587-1604, 2006. 